to appear in Research in Astron. Astrophys. 2012 Vol 12 No. 8, 917-946

http://www.raa-journal.org http://www.iop.org/journals/raa

\title{
The Current Status of Galaxy Formation
}

\author{
Joseph Silk $^{1,2,3}$, Gary A. Mamon ${ }^{1}$ \\ ${ }^{1}$ Institut d'Astrophysique de Paris (UMR 7095: CNRS \& UPMC), 98 bis Boulevard Arago, Paris \\ 75014, France \\ 2 Department of Physics and Astronomy, 3701 San Martin Drive, The Johns Hopkins University, \\ Baltimore MD 21218, USA \\ ${ }^{3}$ Beecroft Institute of Particle Astrophysics and Cosmology, 1 Keble Road, University of Oxford, \\ Oxford OX1 3RH UK
}

Received: 2012 July 12; accepted: 2012 July 17

\begin{abstract}
Understanding galaxy formation is one of the most pressing issues in cosmology. We review the current status of galaxy formation from both an observational and a theoretical perspective, and summarize the prospects for future advances.
\end{abstract}

Key words: galaxy: formation — galaxies: evolution — galaxies: star formation — galaxies: active

\section{INTRODUCTION}

Numerical simulations of large-scale structure have met with great success. However these same simulations fail to account for several of the observed properties of galaxies. On large scales, $0.01-100 \mathrm{Mpc}$, the ansatz of cold, weakly interacting dark matter has led to realistic maps of the galaxy distribution, under the assumptions that light traces mass and that the initial conditions are provided by the observed temperature fluctuations in the cosmic microwave background. On smaller scales, light no longer traces mass because of the complexity of galaxy and star formation. Baryon physics must be added to the simulations in order to produce realistic galaxies. It is here that the modelling is still inadequate.

In this review, we will begin with the standard phenomenology of galaxy formation, then discuss methods and present the recent observational and modeling advances, finishing with a summary of the numerous outstanding issues in galaxy formation theory.

\section{PHENOMENOLOGY}

\subsection{The luminosity function}

Theory provides the mass function of dark halos. Observation yields the luminosity function of galaxies, usually fit by a Schechter (1976) function. Comparison of the two is at first sight disconcerting. One can calculate the $M / L$ ratio for the two functions to overlap at one point, for a mass $M^{*}$ corresponding to $L_{*}$. Define $t_{\text {cool }}=3 / 2 n k T /\left[n^{2} \Lambda(T)\right]$ and $t_{\mathrm{dyn}}=3 /(\sqrt{32 \pi G \rho})$. For star formation to occur, cooling is essential, and the condition $t_{\text {cool }}<t_{\text {dyn }}$ guarantees cooling in an inhomogeneous galactic halo where gas clouds collide at the virial velocity. One finds that

$$
M_{\mathrm{cool}}^{*}=\frac{\alpha^{3}}{\alpha_{g}^{2}} \frac{m_{p}}{m_{e}} \frac{t_{\mathrm{cool}}}{t_{\mathrm{dyn}}} T^{1+2 \beta},
$$




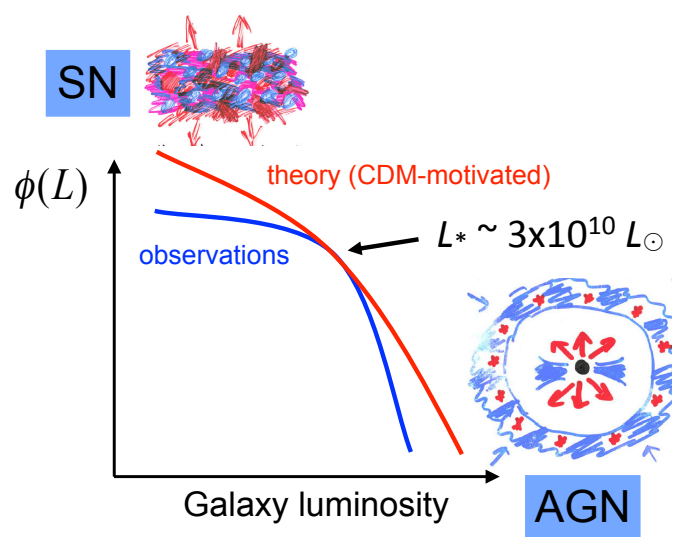

Fig. 1 Role of feedback in modifying the galaxy luminosity function

where $\alpha=e^{2} /(\hbar c)$ and $\alpha_{g}=G m_{p}^{2} / e^{2}$ are the electromagnetic and gravitational fine structure constants. For a cooling function $\Lambda(T) \propto T^{\beta}$, over the relevant temperature range $\left(10^{5}-10^{7} \mathrm{~K}\right)$, one can take $\beta \approx-1 / 2$ for a low metallicity plasma (Gnat \& Sternberg, 2007). The result is that one finds a characteristic galactic halo mass, in terms of fundamental constants, to be of order $10^{12} \mathrm{M}_{\odot}$ (Silk, 1977). The inferred value of the mass-to-light ratio $M / L$ is similar to that observed for $L_{*}$ galaxies. This is a success for theory: dissipation provides a key ingredient in understanding the stellar masses of galaxies, at least for the "typical" galaxy. The characteristic galactic mass is understood by the requirement that cooling within a dynamical time is a necessary condition for efficient star formation (Fig. 1].

However, the naïve assumption that stellar mass follows halo mass, leads to too many small galaxies, too many big galaxies in the nearby universe, too few massive galaxies at high redshift, and too many baryons within the galaxy halos. In addition there are structural problems: for example, massive galaxies with thin disks and/or without bulges are missing, and the concentration and cuspiness of cold dark matter is found to be excessive in barred galaxies and in dwarfs. The resolution to all of these difficulties must lie in feedback. There are various flavors of feedback that span the range of processes including reionization at very high redshift, supernova ( $\mathrm{SN}$ ) explosions, tidal stripping and input from active galactic nuclei (AGN). All of these effects no doubt have a role, but we shall see that what is missing is a robust theory of star formation as well as adequate numerical resolution to properly model the interactions between baryons, dynamics and dark matter.

\subsection{Star formation rate and efficiency}

In addressing star-forming galaxies, the problem reduces to our fundamental ignorance of star formation. Phenomenology is used to address this gap in our knowledge. Massive star feedback in giant molecular clouds, the seat of most galactic star formation, implies a star formation efficiency (SFE), defined as star formation rate (SFR) divided by the ratio of gas mass to dynamical or disk rotation time, of around $2 \%$. This is also found to be true globally in the Milky Way (MW) disk.

Remarkably, a similar SFE is found in nearby star-forming disk galaxies. Indeed, SFRs per unit area in disk galaxies, both near and far, can be described by a simple law, with SFE being the controlling parameter (Silk, 1997; Elmegreen, 1997):

$$
\mathrm{SFE}=\frac{\mathrm{SFR} \times \text { DYNAMICAL TIME }}{\text { GAS MASS }} \approx 0.02 .
$$


The motivation comes from the gravitational instability of cold gas-rich disks, which provides the scaling, although the normalization depends on feedback physics. For the global law, in terms of SFR and gas mass per unit area, SN regulation provides the observed efficiency of about $2 \%$ which fits essentially all local star-forming galaxies. One finds from simple momentum conservation that $\mathrm{SFE}=\sigma_{\text {gas }} v_{\text {cool }} m *_{\mathrm{SN}} / E_{\mathrm{SN}}^{\text {initial }} \approx 0.02$. Here, $v_{\text {cool }}$ is the SN-driven swept-up shell velocity at which approximate momentum conservation sets in and $m *_{\mathrm{SN}} \approx 150 \mathrm{M}_{\odot}$ is the mass formed in stars per SNII, in this case for a Chabrier (2003) initial mass function (IMF). This is a crude estimator of the efficiency of SN momentum input into the interstellar medium, but it reproduces the observed global normalization of the star formation law.

The fit applies not only globally but to star formation complexes in individual galaxies such as M51 and also to starburst galaxies. The star formation law is known as the Schmidt-Kennicutt law (Kennicutt et al. 2007), and its application reveals that molecular gas is the controlling gas ingredient. In the outer parts of galaxies, where the molecular fraction is reduced due to the ambient UV radiation field and lower surface density, the SFR per unit gas mass also declines (Bigiel et al., 2011).

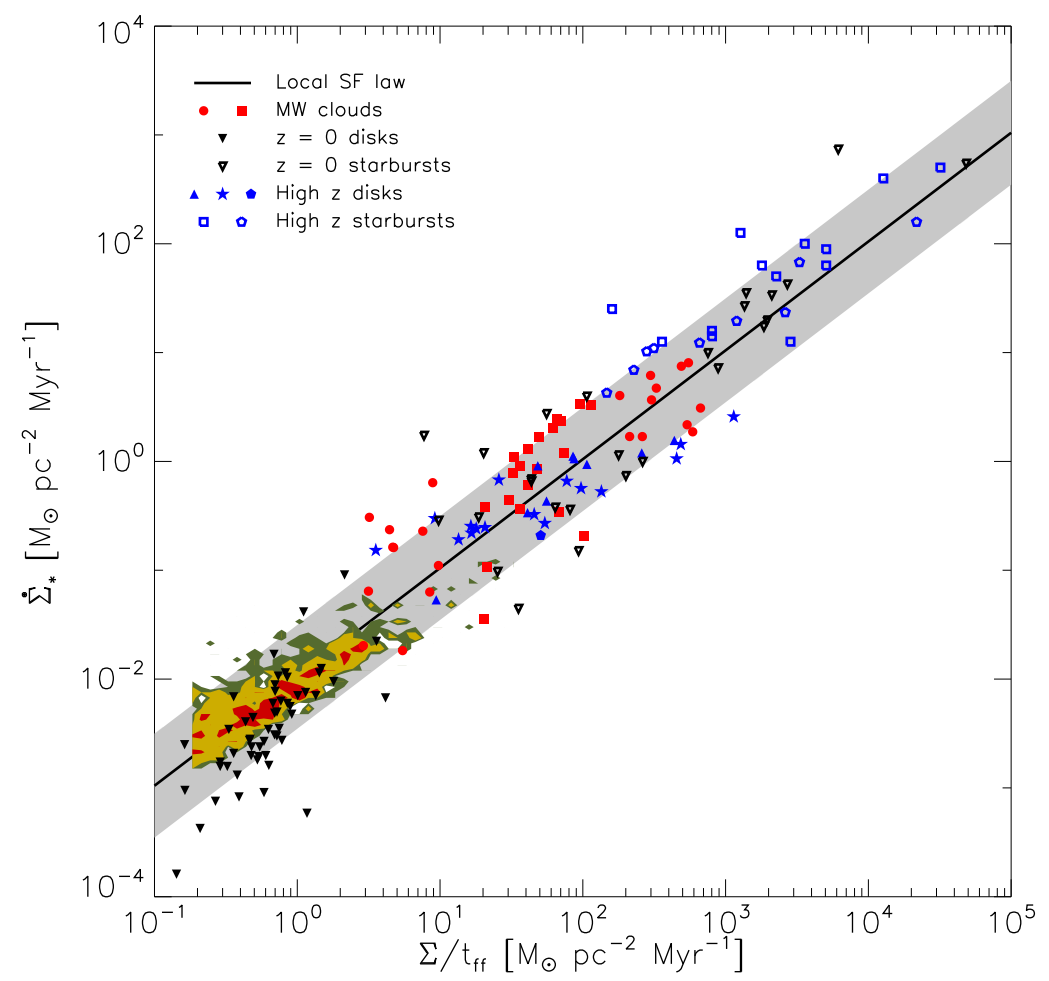

Fig. 2 Schmidt-Kennicutt laws on nearby (including Local Group galaxies as shaded regions) and distant galaxies, as well as Milky Way Giant Molecular Clouds (Krumholz et al., 2012). The solid line is similar to equation (1).

For disk instabilities to result in cloud formation, followed by cloud agglomeration and consequent star formation, one also needs to maintain a cold disk by accretion of cold gas. There is ample evidence of a supply of cold gas, for example in the M33 group. Other spiral galaxies show extensive reservoirs of HI in their outer regions, for example NGC 6946 (Boomsma et al. 2008) and UGC 2082 (Heald et al. 
2011). Recent data extends the Schmidt-Kennicutt law to $z \sim 2$, with a tendency for ultraluminous starbursts at $z \sim 2$ to have somewhat higher SFE (Genzel et al., 2010, see Fig. 2).

A more refined theoretical model needs to take account of star formation in a multi-phase interstellar medium. One expects self-regulation to play a role. If the porosity in the form of SN remnant-driven bubbles is low, there is no venting and the pressure is enhanced, clouds are squeezed, and SN explosions are triggered by massive star formation. This is followed by high porosity and blow-out, and the turbulent pressure drops. Eventually halo infall replenishes the cold gas, the porosity is lowered and the cycle recommences. Some of this complexity can be seen in numerical simulations (Agertz et al., 2011). SNe provide recirculation and venting of gas into fountains, thereby reducing the SFE and prolonging the duration of star formation in normal disk galaxies.

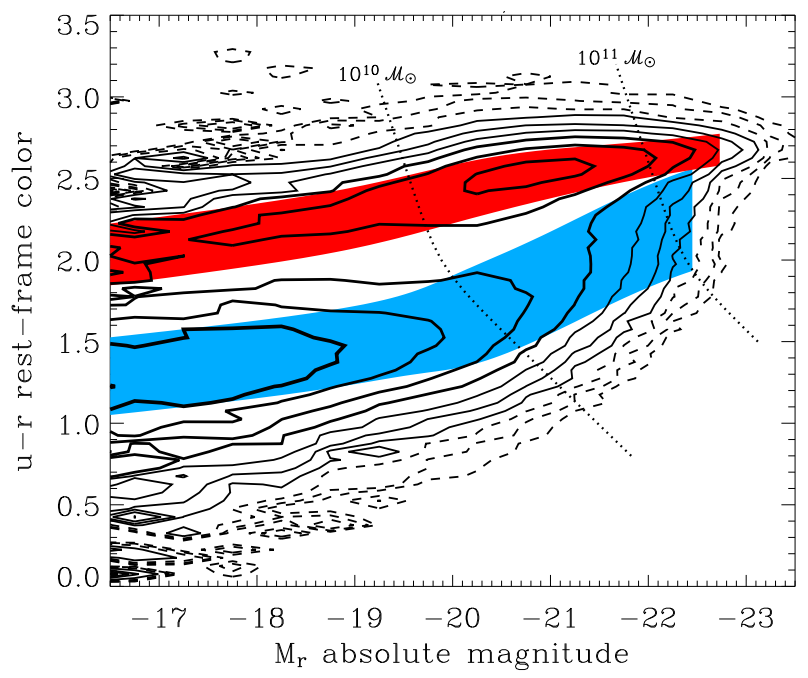

Fig. 3 Illustration of galaxy bimodality. The contours are the density of SDSS galaxies in color-luminosity space, after correction for selection effects (Baldry et al., 2004).

In fact, galaxy colors illustrate the bimodality of SFRs. Elliptical and lenticular galaxies are red, spirals are blue. This lyric does not hide a continuity in galaxy properties: most galaxies lie in either the Red Sequence or the Blue Cloud (see Fig. 3). This suggests that star formation in galaxies is either ongoing or was quenched several Gyr ago. The small fraction of intermediate population, Green Valley galaxies suggests that some galaxies have experienced a recent quenching of their star formation. Seyfert galaxies have intermediate age stellar populations (Schawinski et al., 2007, see fig. 4) and mostly lie in the Green Valley (Schawinski, 2012). This suggests that star formation is quenched by nuclear activity.

\subsection{Scaling relations}

The global properties of early-type galaxies are known to correlate: early work focussed on $L \sim \sigma_{v}^{4}$ (Faber \& Jackson, 1976). The early work found a slope of 4 because of the inclusion of bright and faint galaxies. The modern work finds a slope of 5 for luminous galaxies $\left(M_{B} \lesssim-20.5\right.$, core-Sérsic galaxies) and a slope of 2 for the less luminous spheroids, and has been distilled into the Fundamental Plane linking mass, mass-to-light ratio, and mean surface brightness at the effective radius (Bender et al. 1992). (Bender et al. 1992).

Figure 5 shows a more modern version of the properties of early-type galaxies, to which are added globular clusters and clusters of galaxies. It is not yet understood what makes the continuity of the 


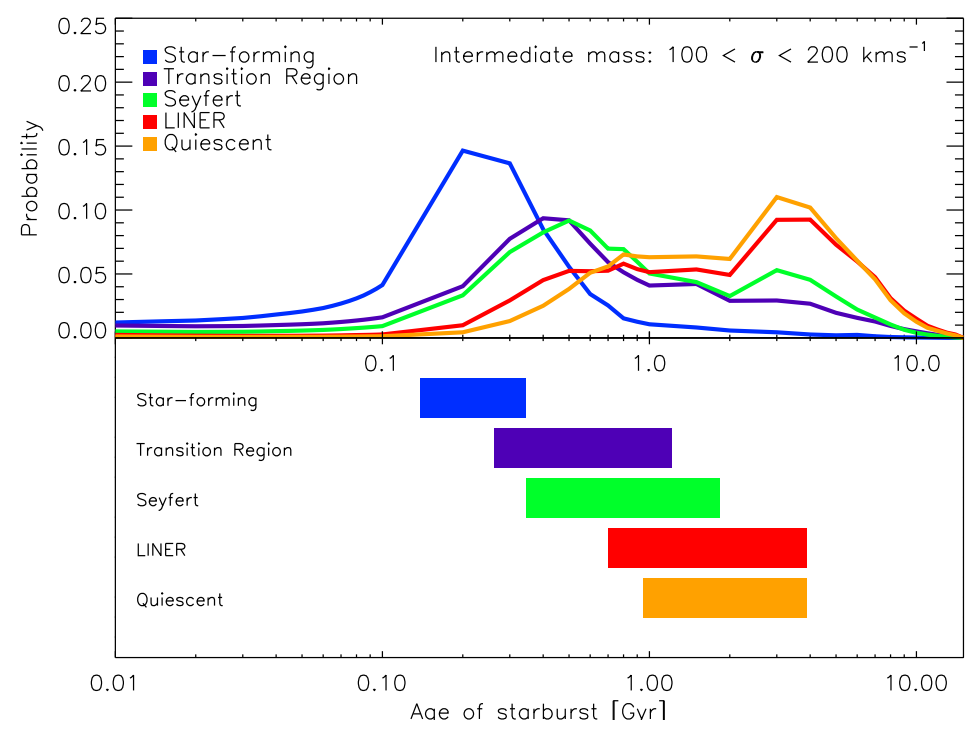

Fig. 4 Ages of galaxies of different activity (Schawinski et al., 2007)

global properties of massive systems fragment into two branches with ultra-compact dwarfs and globular clusters on one side and dwarf spheroidals on the other.

\subsection{Evolution of low mass galaxies}

The accepted solution for gas disruption and dispersal in intermediate mass and massive dwarfs (halo mass $\sim 10^{8}-10^{10} \mathrm{M}_{\odot}$ ) is by $\mathrm{SN}$ feedback. SNe expel the remaining baryons in systems of halo mass up to $\sim 10^{8} \mathrm{M}_{\odot}$, leaving behind dim remnants of dwarf galaxies (Dekel \& Silk. 1986). Presumably the luminous dwarfs accrete gas at later epochs. Most gas is ejected by the first generations of SNe for systems with escape velocity $\lesssim 50 \mathrm{~km} / \mathrm{s}$, leaving dim stellar remnants behind.

In very low-mass halos gas cannot even fall in, because its specific entropy is too high (Rees. 1986). This entropy barrier amounts to a temperature barrier since the gas density, which to first order is proportional to the total mass density, is the same in different halos at a given epoch. Only halos of mass $\gtrsim 10^{5} \mathrm{M}_{\odot}$ trap baryons that are able to undergo early $\mathrm{H}_{2}$ cooling and eventually form stars. Hydrodynamical simulations indicate that this lower limit is sharp (Gnedin, 2000, Okamoto et al., 2008). Reionization reinforces this limit by heating the intergalactic gas to high entropy, hence suppressing subsequent star formation (see Fig. 6). The abrupt increase of the sound speed to $\sim 10-20 \mathrm{~km} / \mathrm{s}$ at $z \sim 10$ means that dwarfs of halo mass $\sim 10^{6}-10^{7} \mathrm{M}_{\odot}$, which have not yet collapsed and fragmented into stars, will be disrupted. However massive dwarfs are unaffected, as are the high $\sigma$ peaks that develop into early collapsing, but rare, low mass dwarfs.

\subsection{Specific SFR}

Other serious, not unrelated, problems arise with low mass galaxies. In the hierarchical approach, these generically form early. Theoretical models, both SAMs and hydrodynamical, appear to fail to account for the observed specific star formation rates (SFR per unit stellar mass or SSFR, Weinmann et al. . 2012), producing too little star formation at late times. Metallicity-dependent star formation alleviates the high redshift problem, reducing the stellar mass that is in place early and enhancing the SSFR as needed 


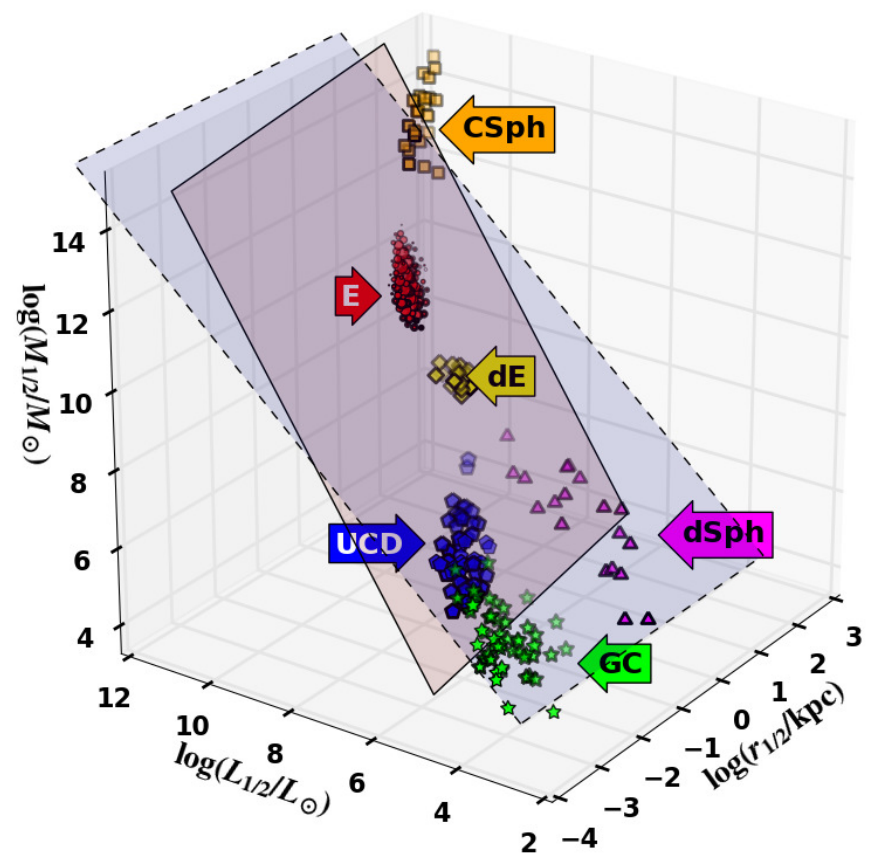

Fig. 5 3D view of scaling relations of spheroidal systems from globular clusters (GC) to clusters of galaxies (CSph), via ultra-compact dwarfs (UCD), dwarf spheroidals (dSph), dwarf ellipticals (dE) and giant ellipticals (E), where the axes are half-luminosity, half-luminosity radius and total mass within half-luminosity radius (Tollerud et al., 2011). The red and blue planes respectively represent the Fundamental Plane and the "virial plane" of constant $M / L$.

(Krumholz \& Dekel, 2012). However, it leads to inconsistency at low redshift, because the change in metallicity and the gas fraction anti-correlate, hence leading to too little evolution in the SSFR.

As shown in Fig. 7, the star formation time-scale (or 1/SSFR) goes from the MW value of $\sim 10 \mathrm{Gyr}$ at low redshift to $\sim 0.5 \mathrm{Gyr}$ at $z \gtrsim 2$. This result suggests two distinct feedback-regulated modes of star formation: at low redshift via SNe and without AGN, and at high redshift with, most plausibly, quenching and possibly triggering by AGN playing a central role. One would expect a transition between these two modes as the AGN duty cycle becomes shorter beyond $z \sim 1$.

A related triggering mechanism appeals to enhanced rate of merging at high $z$ (Khochfar \& Silk. 2011). Alternatively, it has been argued that intensified halo cold gas accretion at early epochs may account for all but the most the extreme SFRs at high $z$, although this may require an implausibly high SFE (Dekel et al. 2009).

\subsection{Spheroidal galaxies}

The baryon fraction is far from its primordial value in all systems other than massive galaxy clusters. $\mathrm{SNe}$ cannot eject significant amounts of gas from massive galaxies. Baryons continue to be accreted over a Hubble time and the stellar mass grows. One consequence is that massive galaxies are overproduced in the models, and that the massive galaxies are also too blue.

Galaxies like the MW have peanut-shaped pseudobulges, in contrast with the classical bulges of more massive spirals. If formed by secular gas-rich disk instabilities, they should have an age distribution 


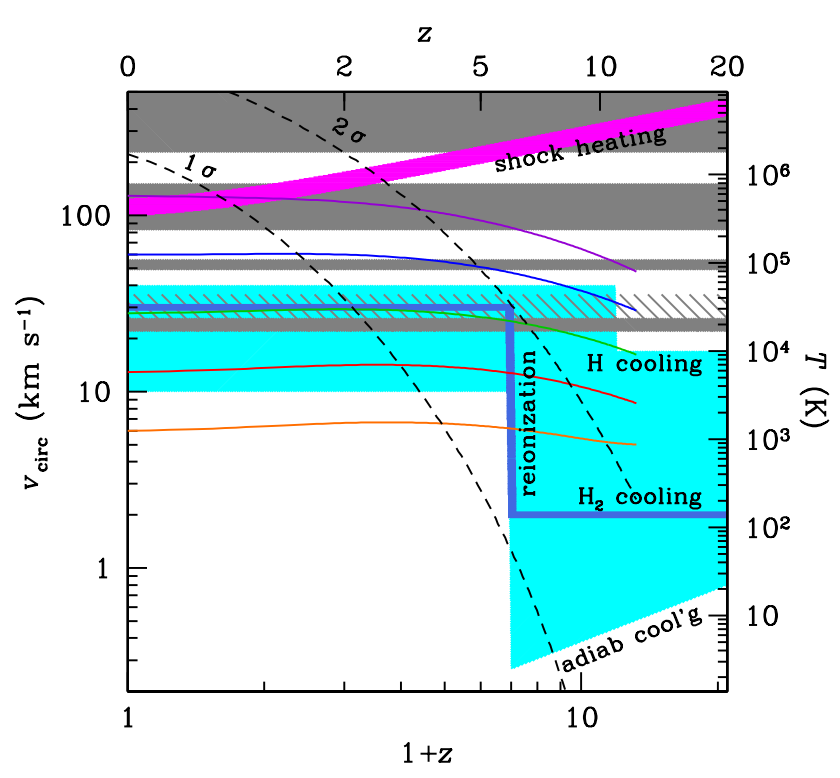

Fig. 6 Evolution of circular velocities (at virial radius) for halos with efficient star formation (Mamon et al., 2012). The smooth curves indicate the mean evolution of halos (with final masses $\log h M=8$ to 12 going upwards). The blue broken line is a model for the evolution of the minimum mass for galaxy formation (set by entropy feedback and related to the temperature of the IGM) and the cyan shaded region represents our ignorance of this parameter. The magenta curve is the maximum circular velocity for efficient gas infall. The grey shaded bands represent regions of thermal instability. The dashed curves shows 1 and $2 \sigma$ fluctuations from the $\Lambda \mathrm{CDM}$ primordial density fluctuation spectrum.

similar to that of the old disk. However the formation time would be at least $\sim 1$ Gyr. The elevated $\alpha /[\mathrm{Fe}]$ ratio of our bulge favors a shorter formation time. This would be more consistent with an early disk instability phase reminiscent of that associated with clumpy gas-rich galaxies observed at $z \sim$ 2. Massive clump merging provides a possible solution for forming bulges at a relatively late epoch (Ceverino et al., 2010).

However the time-scale (several Gyr) is too long to result in the enhanced $\alpha /[\mathrm{Fe}]$ ratios characteristic of massive spheroids, or even the less extreme enhancement in the MW bulge. The shorter timescales required arise in more plausible cosmological initial conditions that result in a redshift $z>2$ for pseudobulge formation (Okamoto, 2012).

\subsection{The role of AGN}

SNe have little impact on the formation of massive galaxies. Feedback from SN explosions fails to stop the streaming of cold flows towards the centre (Powell et al., 2011). The SN ejecta tends to be driven out with only modest interaction with, and entrainment of, cold infalling gas. A more coherent and effective interaction is provided by AGN feedback from supermassive black holes (SMBH). A clue towards a solution for these dilemmas comes from the accepted explanation of the Magorrian et al. relation, which relates SMBH mass to spheroid mass (Magorrian et al. 1998) and velocity dispersion (Ferrarese \& Merritt, 2000, see Fig. 8. 

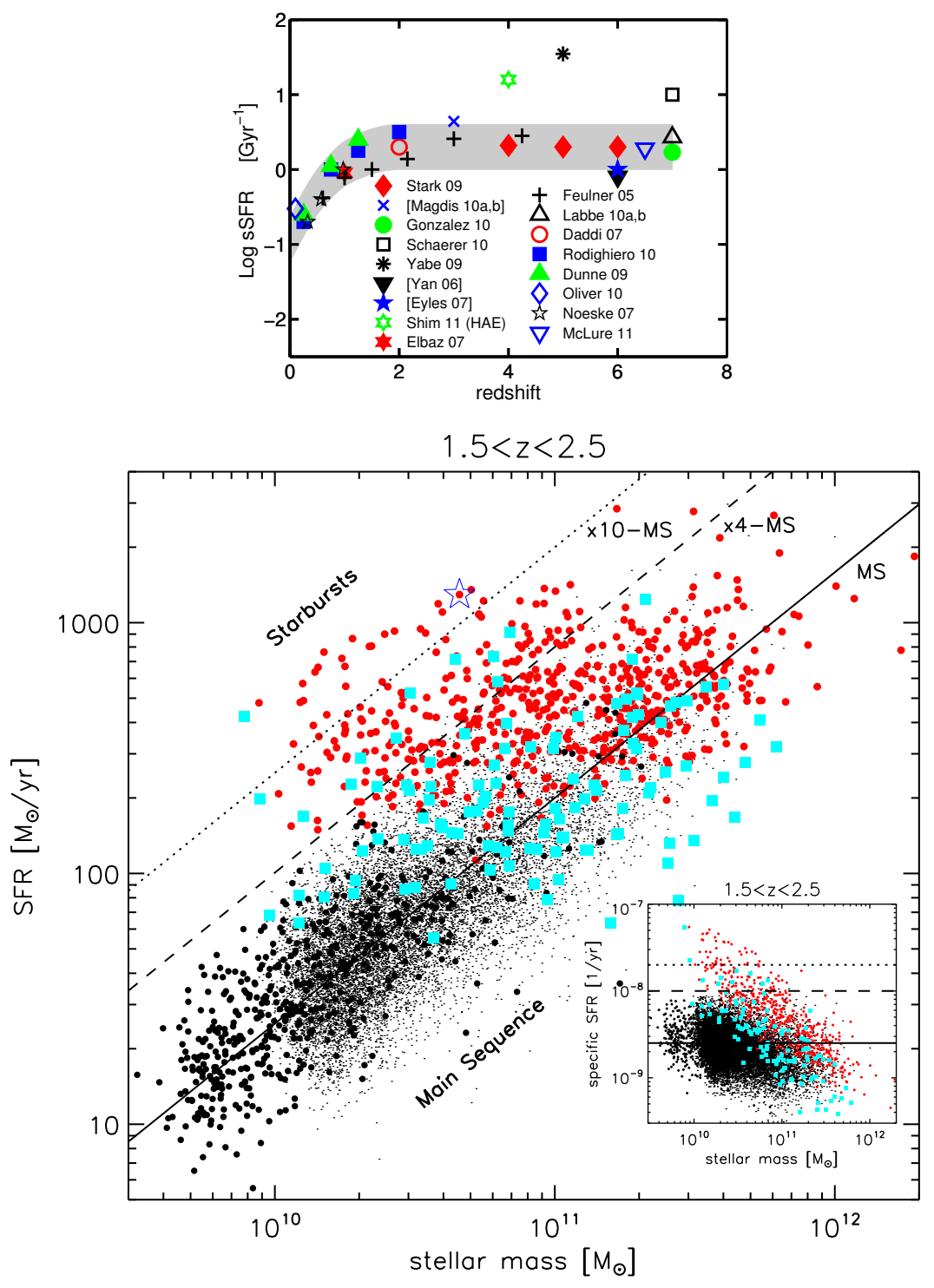

Fig. 7 Evolution of the specific SFR (SSFR) of galaxies of stellar mass $0.2-1 \times 10^{10} \mathrm{M}_{\odot}$ (Weinmann et al. 2011, top); SFR for galaxies from different samples (different color symbols), highlighting the "Main Sequence" and a population of starbursts, with SSFR massdependence inset (Rodighiero et al. 2011, bottom).

This requires collusion between black hole growth and the initial gas content of the galaxy when the old stellar spheroid formed. One conventionally appeals to outflows from the central black hole that deliver momentum to the protogalactic gas. When the black hole is sufficiently massive, the Eddington luminosity is high enough that residual gas is ejected. An estimate of the available momentum supply come from equating the Eddington momentum with self-gravity on circumgalactic gas shells, $L_{\mathrm{Edd}} / c=$ $4 \pi G M / \kappa=G M M_{\text {gas }} / r^{2}$, where $\kappa$ us the opacity. Blowout occurs and star formation terminates when the SMBH- $\sigma_{v}$ relation saturates. This occurs for $M_{\mathrm{BH}} \propto \sigma_{v}^{4}$, close to the observed slope of $\gtrsim 5$ 


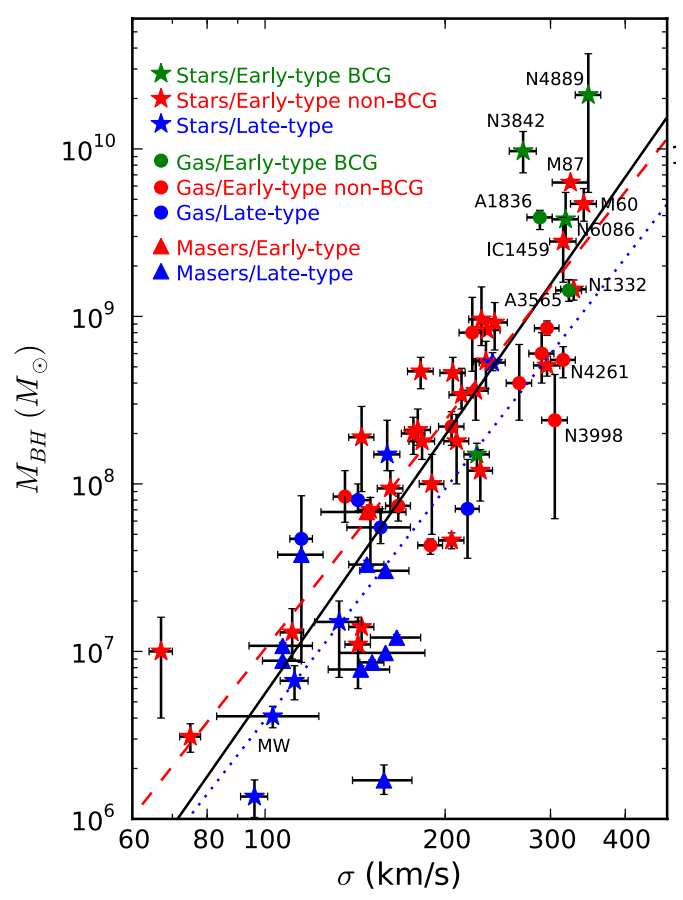

Fig. 8 Black hole mass versus spheroid velocity dispersion (luminosity-weighted within one effective radius), from McConnell et al. (2011)

(Graham et al. 2011), and gives the correct normalization of the relation, at least in order of magnitude. This is the early feedback quasar mode.

There is also a role for AGN feedback at late epochs, when the AGN radio mode drives jets and cocoons that heat halo gas, inhibit cooling, resolve the galaxy luminosity function bright end problem and account for the red colors of massive early-type galaxies. AGN feedback in the radio mode may also account for the suppression in numbers of intermediate mass and satellite galaxies (e.g., Cattaneo et al. 2009 and references therein). Feedback from AGN in the host galaxies also preheats the halo gas that otherwise would be captured by satellites.

\subsection{Galaxies downsize}

Our understanding of galaxy formation is driven by observations. Prior to 2000 or so, it was accepted that hierarchical galaxy formation predicted that small galaxies form prior to massive galaxies. The first indications that this was in error came from the recognition that more massive early-type galaxies have redder colors (de Vaucouleurs, 1961), higher metallicities (Faber. 1973) and enhanced $[\alpha] /[\mathrm{Fe}]$ metallicity ratios (Ziegler et al. 2005), indicative of an older stellar population with a shorter star formation time (see Fig. 9). This effect is called downsizing, as the most massive galaxies have their stellar populations in place early. In effect, we have a cosmic clock: incorporation into stars of debris from SNe II ( $\lesssim 10^{8}$ yr) versus SNe I ( $\gtrsim 10^{9} \mathrm{yr}$ ) provides a means of dating the duration of star formation. This result was soon followed by infrared observations that showed that stellar mass assembly favored more massive systems at earlier epochs (González et al. 2011a). 

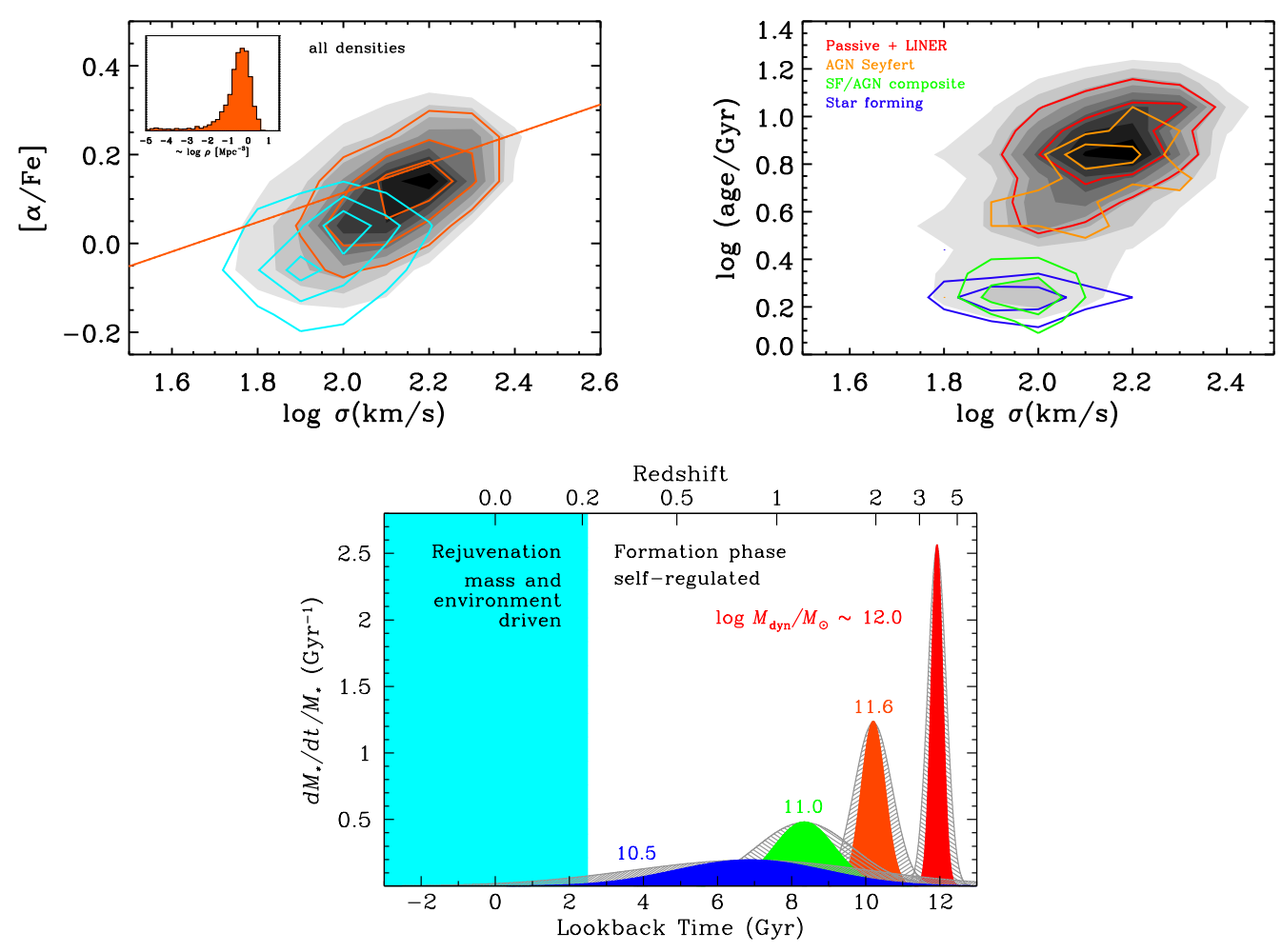

Fig. 9 Metallicity ratio and age versus galaxy velocity dispersion (i.e. mass) and history of star formation (Thomas et al., 2010)

\subsection{Morphological evolution}

We cannot do justice in this review to a largely phenomnological discussion of morphological evolution of both disk and irregular galaxies. Here, observations are far ahead of theory. However, there are strong arguments to support a continuous sequence between dwarf spheroidal galaxies and S0 galaxies (Kormendy \& Bender. 2012). The transformation applies to the disk components and may involve ram pressure stripping of cold gas (Gunn \& Gott, 1972) as well as galaxy harrassment (Moore et al., 1998). This sequence seems to acts in parallel to the pseudobulges or bulges of S0 galaxies being generated via stripped/harassed or simply starved disk galaxies (Kormendy \& Bender. 2012).

\section{METHODS}

\subsection{Observational surveys}

The fundamental driver of progress in astronomy is through observations. The advent of large galaxy surveys, either wide spectroscopic surveys probing the nearby Universe (e.g., SDSS) or narrower surveys using photometric redshifts and often in the infrared domain (e.g., with Spitzer and Herschel) to probe distant galaxies in the optical and near-infrared domains, has led to formidable progress in understanding galaxy formation. Nevertheless, it is difficult to link the galaxies we see at high redshift with the ones we see in local Universe, and one is prone to Malmquist bias, as well as aperture and other selection effects. 


\subsection{Semi-Analytical Models}

Several simulation techniques have been developed to be able to link galaxies from the past to the present, and to obtain a statistical view of the variety of the evolution histories of galaxies, in terms of star formation, stellar mass assembly and halo mass assembly.

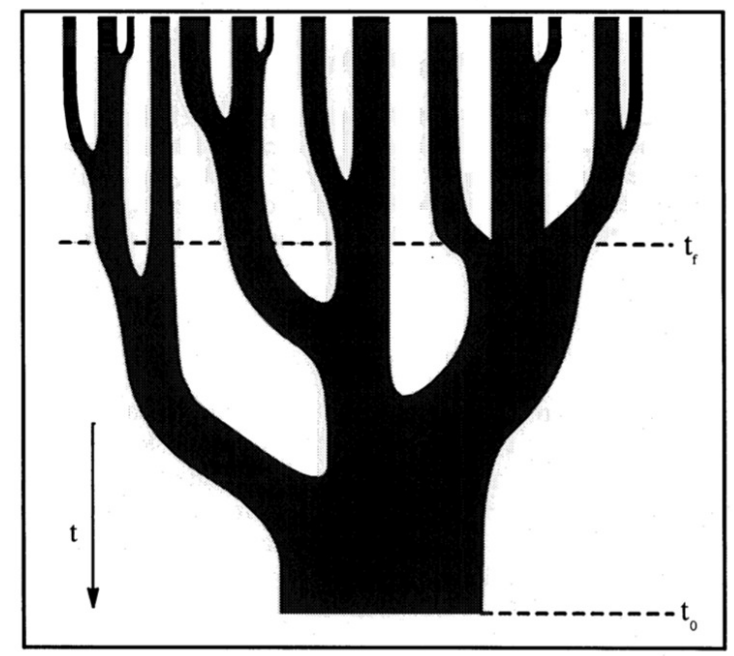

Fig. 10 Illustration of halo merger tree (Lacey \& Cole 1993) showing the progenitors of a halo selected at time $t_{0}$

Given current computational constraints, it is impossible to achieve the sub-parsec or finer resolution needed to adequately model star formation and accretion onto black holes in a cosmological simulation. Theorists have invented a swindle, wherein the complex processes of star formation and accretion onto SMBH are hidden inside a black box called "sub-grid physics" that can be tagged onto a large-scale simulation. In SAMs, galaxies are "painted" on halos built from halo merger trees or detected in cosmological dissipationless (dark matter only) simulations. The former (see Fig. 10p produce the mass assembly history (MAH) of halos with the condition that they end up in a halo of mass $M_{0}$ at epoch $z_{0}$ (usually $z_{0}=0$ ). The branches are drawn from random samplings given the known conditional probabilities arising from extensions (Bower, 1991, Lacey \& Cole, 1993) and modifications of the Press \& Schechter (1974) formalism. Halo merger trees have the advantage of being rapid to compute, but lack positional information. Cosmological simulations, with up to $10^{8}$ particles, are becoming increasingly common, but are intensive to process, in particular to detect halos (Knebe et al., 2011 and references therein) and subhalos (Onions et al., 2012 and references therein) and build halo merger trees.

Once the halo MAH is identified, one follows the branches of the tree from past to present, to build galaxies. The galaxy formation recipe includes several ingredients:

1. The gas cooling time must be short for the gas to dissipatively cool into a disk. In particular, gas cannot fall onto low-mass halos because of the cooling barrier and falls less efficiently onto highmass halos because of a virial shock, whereas gas can infall along cold filaments on lower mass halos.

2. Star formation occurs at a rate $\dot{m}=\operatorname{cst} m_{\mathrm{gas}} / t_{\mathrm{dyn}}$, where $t_{\mathrm{dyn}}$ is a measure of the dynamical time of the galaxy.

3. Feedback from $\mathrm{SNe}$ and from the relativistic jets arising from central SMBHs hiding as AGN heats up the surrounding gas. 
4. While the gas settles into disks, major mergers of galaxies cause disks to transform into ellipticals, and after subsequent disk build-up, the merger remnant is identified to a bulge inside a spiral galaxy. The bulge can also be built-up by repeated minor mergers, as well as starbursts and secular evolution of the disk.

5. The SAM keeps track of star formation times and predicts galaxy luminosities in different wavebands using population synthesis codes.

6. When a smaller halo enters a larger one, it becomes a subhalo, its galaxy becomes a satellite, and usually, the gas that attempts to fall onto the subhalo is now directed towards the central galaxy of the halo.

7. Satellite galaxy trajectories are assumed to be those of the subhalos they belong to, and when they are no longer resolved in the cosmological simulation, or if one is only using a halo merger tree, the galaxies are merged with the central galaxy on a dynamical friction timescale (calibrated on simulations, e.g. Jiang et al., 2008).

An advantage of SAMs is that it is easy to gauge the importance of various physical processes by seeing how the outcome is changed when a process is turned off in the SAM.

Present-day SAMs are increasingly complex, and SAMs can include up to $10^{5}$ lines of code. The popularity of SAMs has increased with public-domain outputs (Bower et al., 2006, Croton et al., 2006, De Lucia \& Blaizot, 2007, Guo et al., 2011) and codes (Benson, 2012).

\subsection{Hydrodynamical simulations}

The weakness of SAMs is that much of the physics is controlled by hand (except for gravity, when the SAMs are directly applied to cosmological N-body simulations of the dark matter component). Hydrodynamical simulations provide the means to treat hydrodynamical processes in a much more selfconsistent manner, and cosmological hydrodynamical simulations have been run for nearly 25 years (starting from Evrard, 1988).

These simulations come in two flavors: cell-based and particle-based. It was rapidly realized that cell-based methods could not resolve at the same time the very large cosmological scales and the small scales within galaxies, and the early progress in the field was driven by the Smooth Particle Hydrodynamics (SPH) method (Gingold \& Monaghan, 1977; Monaghan, 1992; Springel, 2010b), in which the diffuse gas is treated as a collection of particles, whereas the physical properties (temperature, metal content, etc.) are smoothed over neighboring particles using a given SPH-smoothing kernel. Despite early successes (comparisons of different hydrodynamical codes by Frenk et al., 1999), SPH methods fail to resolve shocks as well as Rayleigh-Taylor and Kelvin-Helmholtz instabilities (Scannapieco et al., 2012). This has brought renewed popularity to cell-based methods, with the major improvement of resolution within the Adaptive Mesh Refinement (AMR) scheme (Kravtsov et al. 1997; O'Shea et al. 2005, Teyssier, 2002), where cells can be refined into smaller cells following a condition on density or any other physical property. Moreover, schemes with deformable cells (that do not follow the Cartesian grid) were developed 20 years ago, and are now becoming more widely used (e.g., AREPO, Springel, 2010a).

In these hydrodynamical codes, stars can be formed when the gas is sufficiently dense, with a convergent flow and a short cooling time. Current codes do not have sufficient mass resolution to resolve individual stars, so the star particles made from the gas are really collections of stars, with an initial mass function. One can therefore predict how many core-collapse SNe will explode after the star particle forms, and the very considerable SN energy is usually redeposited into the gas by adding velocity kicks to the neighboring gas particles and possibly also thermal energy. Similarly, AGN can be implemented, for example by forcing a Magorrian type of relation between the SMBH mass and the spheroidal mass of the galaxy, and feedback from AGN jets can be implemented in a similar fashion as is feedback from SNe. Hydrodynamical codes are therefore not fully self-consistent, as they include semi-analytical recipes for the formation of stars and the feedback from SNe and AGN. 


\subsection{New methods}

\subsubsection{Analytical models}

The growing complexity of SAM codes (e.g., the publicly-available GALCTICUS code of Benson 2012 contains over 120000 lines of code and involves over 30 non-cosmological parameters with non-trivial values) has led some to seek simpler descriptions of galaxy formation.

One level of simplicity is to parameterize the time derivatives of the different components of galaxies (stars, cold gas, hot gas, dark matter) as linear combinations of these parameters (Neistein \& Weinmann, 2010). But one can go to an even simpler level and characterize the fraction of gas that can cool (Blanchard et al. 1992) or the mass in stars (Cattaneo et al., 2011) as a function of halo mass and epoch. Although such approaches are much too simple to be able to capture the details of galaxy formation, they are sufficient to study simple questions.

Assuming that all the gas in the range of temperatures between $10^{4} \mathrm{~K}$ and the maximum where the cooling time is shorter than the age of the Universe (or the dynamical time) effectively cools, and that the gas is replenished one the timescale where halos grow, Blanchard et al.(1992) showed that nearly all the baryons should have converted to stars by $z=0$. Since this is not observed, this simple calculation shows that feedback mechanisms are required to prevent too high star formation.

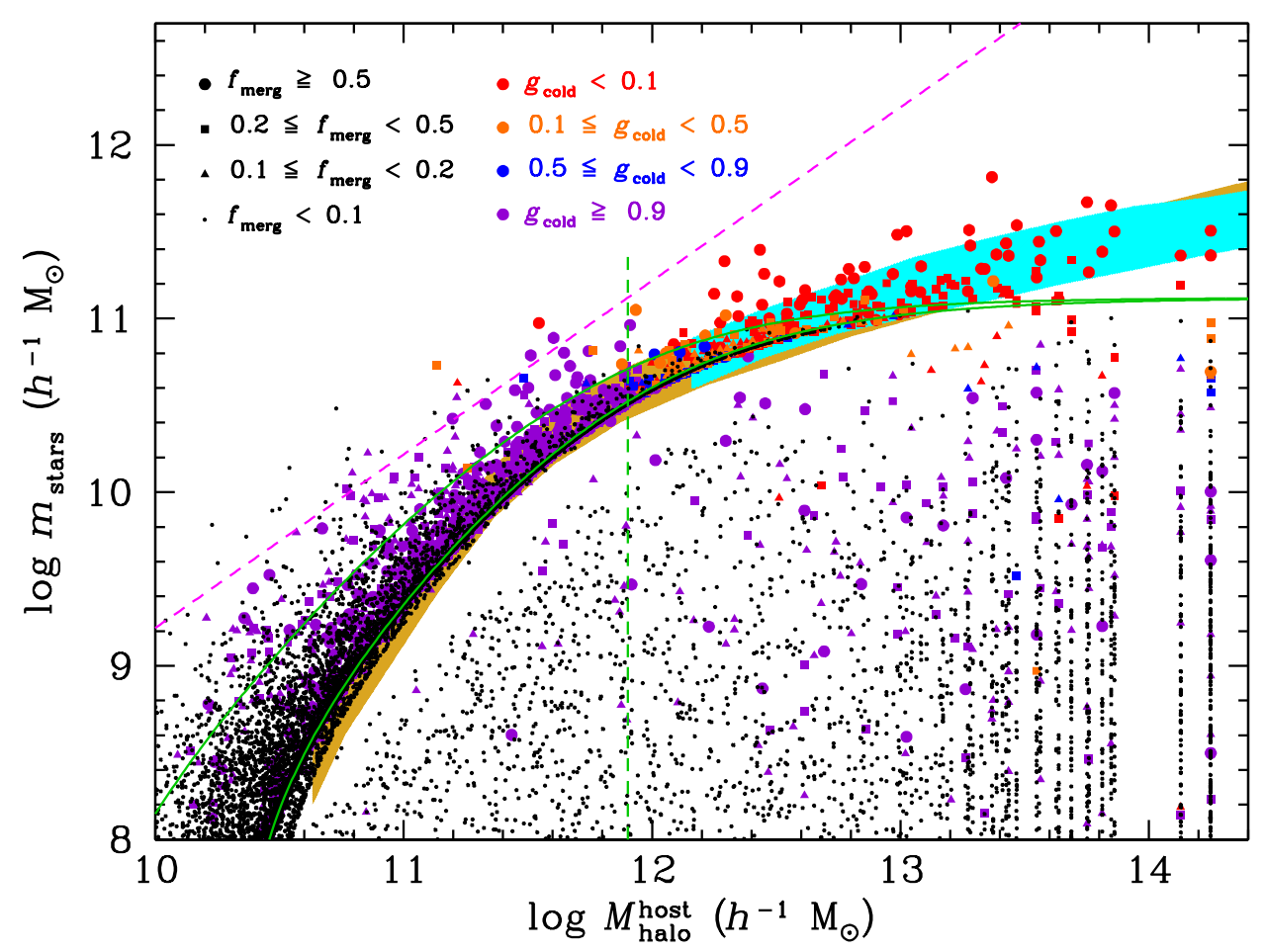

Fig. 11 Stellar versus host halo mass from analytical model by Cattaneo et al. (2011) run on dark matter simulation. The shaded regions are results obtained from the conditional luminosity function (blue, Yang et al., 2009) and abundance matching (gold, Guo et al., 2010). The large symbols denote galaxies who have acquired most of their stellar mass through mergers (rather than smooth gas accretion). The green curves show the galaxy formation model at $z=0$ and $z=3$. 
Cattaneo et al. (2011) apply their simple galaxy formation prescription onto the halos of a highresolution cosmological $N$ body simulation and reproduce the $z=0$ observed stellar mass function with only four parameters, despite the overly simplistic model. They find a fairly narrow stellar versus halo mass relation for the dominant ("central") galaxies in halos and a gap between their stellar masses and those of the satellites, in very good agreement with the relations obtained by Yang et al. (2009) from the SDSS using conditional stellar mass functions (Fig. 11). This gap is remarkable, as it is less built-in Cattaneo et al.'s method than it is in SAMs: for example, halos with two-dominant galaxies (such as observed in the Coma cluster) are allowed. Similar "successful" analytical models have been proposed by Peng et al. (2010) and Bouché et al. (2010).

\subsubsection{Halo Occupation Distribution}

A simple way to statistically populate galaxies inside halos, called Halo Occupation Distribution (HOD) is to assume a functional form for some galaxy statistic in terms of the halo mass. The galaxy statistic can be the multiplicity function (for galaxies more massive or more luminous than some threshold, Berlind \& Weinberg, 2002, see upper left panel of Fig. 12), the luminosity or stellar mass function, generically denoted CLF for conditional luminosity function (Yang et al., 2003). Although these HOD methods have no underlying physics, they are a very useful tool to derive galaxy trends with halo mass, or, in other words to find the effects of the global environment on galaxies.

\subsubsection{Abundance Matching}

An offshoot of HOD models is to link the mean trend of some galaxy property in terms of the mass of its halo, using so-called Abundance Matching (AM). The idea is to solve $N(>x)=N\left(>M_{\text {halo }}\right)$, i.e. matching cumulative distributions of the observed galaxy property, $x$, with the predicted one for halo masses, determined either from theory (Press \& Schechter, 1974, Sheth et al. 2001) or from cosmological $N$ body simulations (Warren et al., 2006; Tinker et al. 2008; Crocce et al. 2010; Courtin et al., 2011).

Common uses of AM involve one-to-one correspondences between 1) stellar and halo mass for central galaxies in halos, 2) total stellar mass and halo mass in halos, and 3) stellar and subhalo mass in galaxies. Marinoni \& Hudson(2002) performed the first such AM analysis to determine $M_{\text {halo }} / L$ versus $L$; they first had to determine the observed cosmic stellar mass function, not counting the galaxies within groups, but only the groups themselves (Marinoni et al. 2002). Guo et al. (2010) used this third approach (called subhalo abundance matching or SHAM, and pioneered independently by Vale \& Ostriker. 2006 and Conroy et al. 2006), to determine the galaxy formation efficiency $m_{\text {stars }} / M_{\text {halo }}$ as a function of $M_{\text {halo }}$, by matching the observed stellar mass function with the subhalo mass function that they determined in the Millennium (Springel et al., 2005) and Millennium-II (Boylan-Kolchin et al., 2009) simulations. Although AM methods are based upon a fine relation between stellar and halo mass, they can easily be adapted to finite dispersion in this relation (Behroozi et al. 2010).

Not only is AM very useful to determine, without free parameters, the relation of stellar to halo mass (lower panel of Fig. 12), but it superbly predicts the galaxy correlation function of SDSS galaxies (Conroy et al. 2006, upper right panel of Fig. 12. The drawback of AM methods is that they do not clarify the underlying physics of galaxy formation.

\section{RESULTS FROM NUMERICAL SIMULATIONS}

\subsection{General results from semi-analytical models of galaxy formation}

SAMs have been remarkably successful in constructing mock catalogs of galaxies at different epochs and are used in motivating and in interpreting the large surveys of galaxies. For example, they reproduce very well the $z=0$ stellar mass function and correlation function (see Fig. 13).

However, attempts to solve the problems of high redshift galaxies have so far been woefully inadequate. For example, they cannot reproduce the rapid decrease in the cosmic SFR since $z=1$ (see 

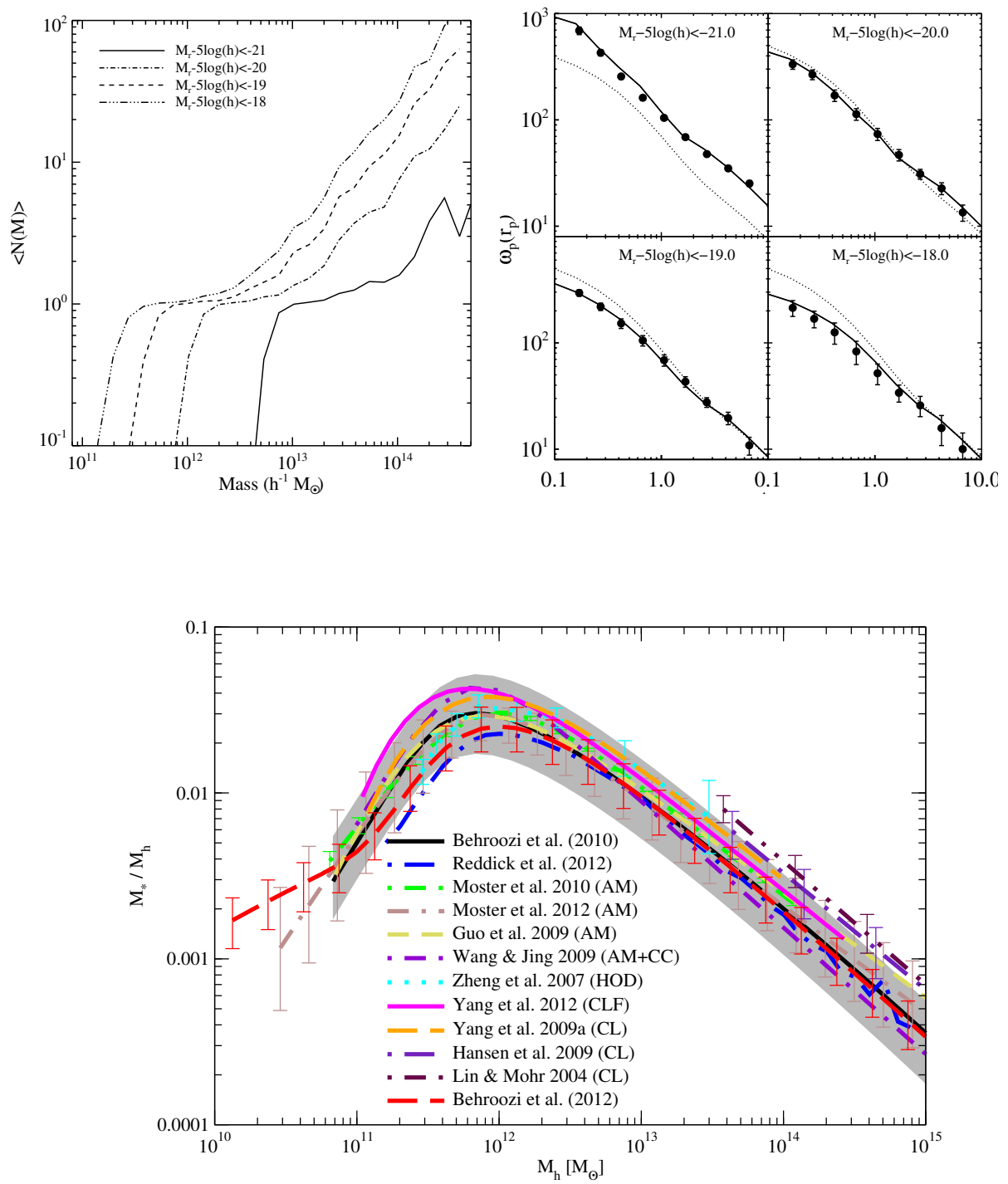

Fig. 12 Top left: Illustration of HOD models of multiplicity functions (per halo) obtained from abundance matching (Conroy et al. 2006). Top right: Abundance matching prediction on the galaxy correlation function compared to SDSS observations (symbols), while the halohalo correlation function is shown as dotted lines (Conroy et al., 2006). Bottom: Halo mass for given stellar mass obtained by abundance matching (AM), HOD, conditional luminosity function (CLF) and group catalogs (CL) at $z=0.1$ (Behroozi et al. 2012). The shaded region shows the AM analysis of Behroozi et al. (2010).

Fig. 14. The early SAM feedback models used AGN quenching, and required excessive dust in early types in the nearby universe (Bower et al. 2006). Refinements to high redshift attempted to account simultaneously for galaxy and AGN accounts, and only succeeded by requiring excessive amounts of dust in order to hide most of the AGNs seen in deep X-ray surveys (Fanidakis et al. 2011). An early 

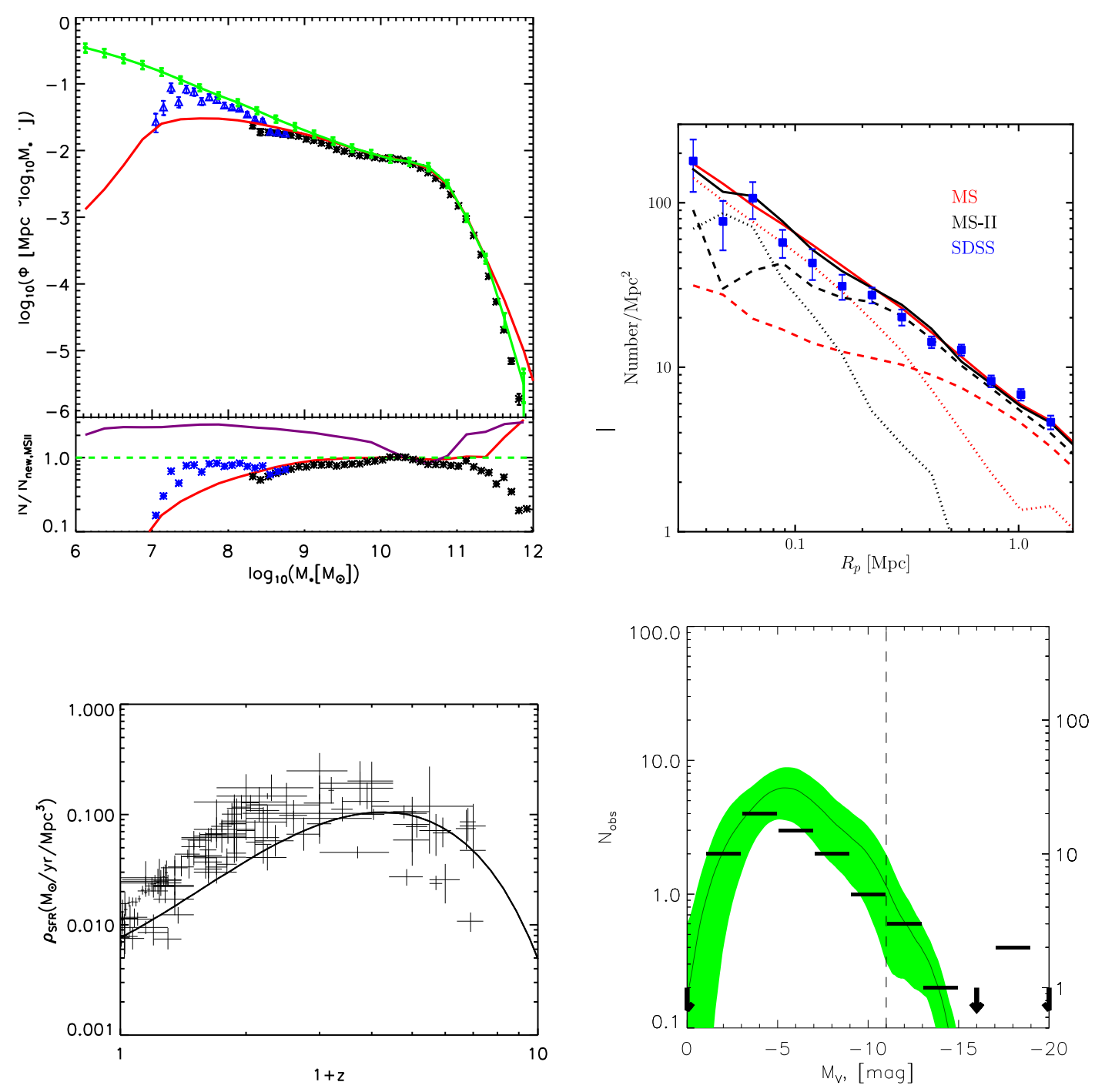

Fig. 13 Illustrations of predictions of SAMs at $z-0$. Upper left: Stellar mass functions (Guo et al. 2011): symbols are from SDSS (Li \& White, 2009), while curves are from a SAM run on both wide and low-resolution Millennium Simulation and on the higher but smaller MS-II simulation. Upper right: Galaxy correlation functions (Guo et al., 2011). Bottom left: Evolution of the cosmic SFR (Guo et al., 2011). Bottom right: Very low end of the galaxy luminosity function (Koposov et al. 2009$)$.

indication that SAMs were entering uncertain territory can be seen in the early predictions of the cosmic star formation history: as numerical resolution was increased, the predicted SFR increased without limit (Springel \& Hernquist, 2003). This makes one begin to doubt the predictive power of SAMs.

Clearly, baryon physics is far more complicated than assumed in the early SAMs of the 1990s. In fact, we still lack an adequate explanation for the evolution of the stellar mass function. Attempts to patch up the problem at low redshift, to avoid an excess of massive galaxies, exacerbate the inadequacy of the predicted numbers of massive galaxies at high redshift (Fontanot et al. 2009). One attempt to 


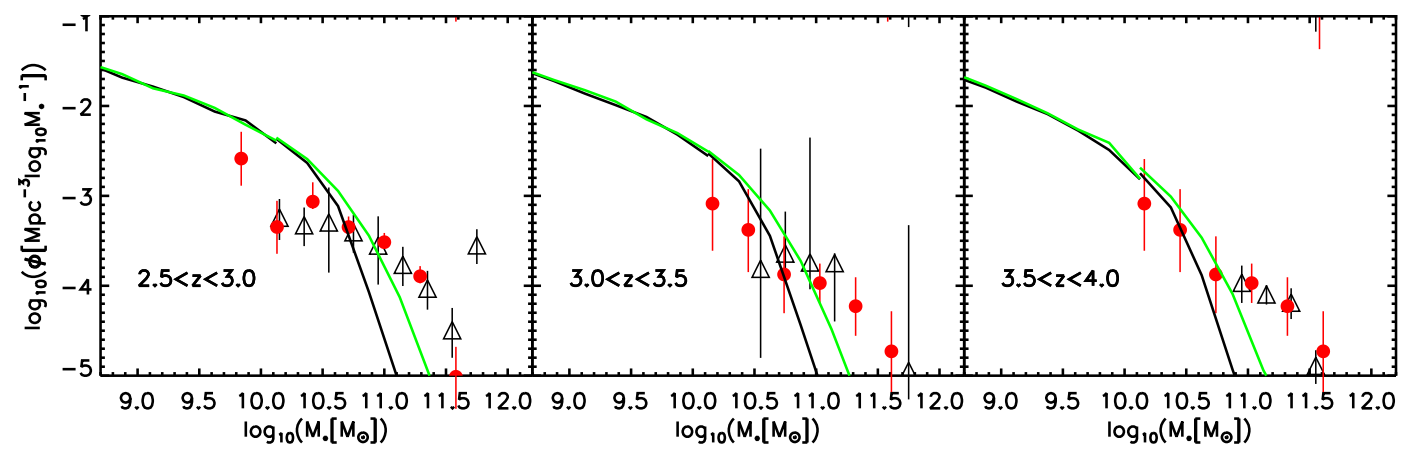

Fig. 14 Evolution of stellar mass functions predicted by Guo et al. (2011). Open triangles and red circles represent observations by Pérez-González et al. (2008) and Marchesini et al. (2009), respectively. Black and green curves represent the predicted stellar mass functions of galaxies, respectively before and after convolving the stellar masses by 0.25 dex measurement errors.

correct the problem at large redshift incorporates for the first time thermally pulsing AGB (or carbon) stars in the models, and the extra NIR luminosity reduces the inferred galaxy masses (Henriques et al. 2011). However the price is that the lower redshift galaxy count predictions no longer fit the models.

\subsection{Feedback and dwarfs}

Dwarf spheroidal galaxies are dark matter laboratories, dominated by dark matter. However the numbers defy interpretation. Feedback is readily adjusted to reduce the numbers of low mass dwarfs (Koposov et al. 2009), but the most massive dwarfs predicted by $\Lambda$ CDM simulations are not observed (BoylanKolchin et al., 2012). This may be a function of the neglect of baryons in the Aquarius simulations: inclusion of baryons reduces the central densities of massive dwarfs (Zolotov et al., 2012). Unorthodox feedback (AGN) may also be a solution (Boylan-Kolchin et al., 2011). Moreover, most low-mass dwarfs have cores rather than the cusps predicted by CDM-only simulations. Baryonic feedback may reconcile data on dwarf core profiles with simulations that include star formation and gas cooling (Oh et al., 2011. Governato et al., 2012), who find that SN-driven outflows help flatten dark matter central density cusps. As mentioned earlier, enhanced early star formation and SN production creates strong tensions with the need for strong late low mass galaxy evolution. SN feedback at later epochs may turn cusps into cores by sloshing of more recently accreted gas clouds (Mashchenko et al., 2006), more recently addressed in Pontzen \& Governato (2012), who consider bulk gas motions and require short intense bursts of star formation. There may be evidence for such phenomena in dwarf galaxies (Weisz et al., 2012).

Multiphase simulations (Powell et al., 2011) confirm the effectiveness of SN-driven winds, but find that they do not lead to baryon ejection. In a multi-phase medium with more realistic filamentary accretion, outflows are only typically $\lesssim 10 \%$ of the gas accretion rate. It is not clear whether SN feedback may still provide enough momentum to yield an acceptable fit to the low mass end of the galaxy luminosity function for the classical dwarfs. Ram pressure stripping (Gunn \& Gott, 1972; Mayer et al. 2007) remains an alternative or complimentary mechanism, and morphological transformation of disks into dwarf spheroidals may be accomplished by repeated rapid encounters, i.e. "harassment" (Moore et al., 1998) or gravitationally-induced resonances (D’Onghia et al., 2009).

SN feedback enables present day disk galaxy properties to be reproduced, including the TullyFisher relation and sizes, except for massive disks. More energetic feedback, from an AGN phase, is envisaged as a possible solution (McCarthy et al., 2012). Many galaxies, including early types, have extended star formation histories. Minor mergers provide an adequate gas supply to account for these (Kaviraj et al., 2009). However, hydrodynamical studies of the baryonic evolution and SFR in low mass 
galaxies disagree about whether or not one can reproduce their observed properties, including dark matter cores and baryon fraction. Outflows may reproduce the observed cores (Governato et al., 2012) if the SFE is high at early epochs, but such models fail to result in the strong evolution observed at low redshift (Weinmann et al., 2012).

Tidal disruption also plays a role in disrupting satellites whose orbits intersect the disk or bulge. Dramatic discoveries due to deep imaging of nearby galaxies with very small, wide field of view, telescopes confirm the ubiquity of tidal tails that trace dwarf disruption in the remote past (MartínezDelgado et al. 2010). Simulations provide a convincing demonstration that we are seeing tidal disruption in action (Cooper et al. 2010). An independent confirmation of disruption in action comes from studies of the tidal tails around the outermost MW globular star clusters such as Pal 13. Gaps in the tails (Grillmair, 2009) indicate the presence of dark satellites. Numerical simulations (Yoon et al., 2011) find that high $M / L$ satellites of mass $\sim 10^{7} \mathrm{M}_{\odot}$ are required, again a prediction of the CDM model.

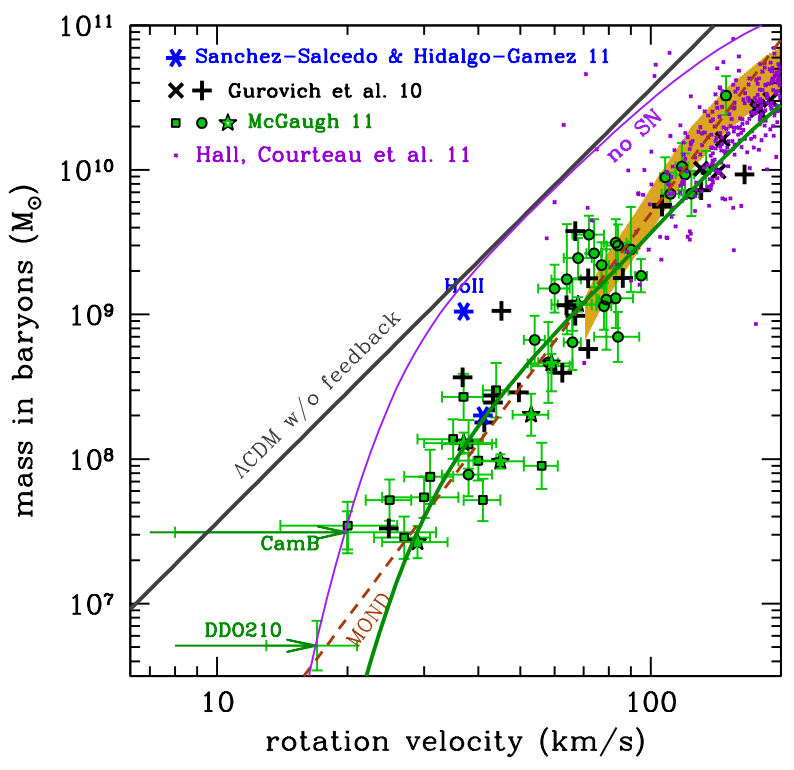

Fig. 15 Baryonic Tully-Fisher relation (Mamon \& Silk, in prep.). Symbols are from HI measurements, where the velocity is the flat part of the rotation curve (green, from McGaugh. 2012) or from line-widths (black, Gurovich et al. 2010, magenta, Hall et al., 2011). The grey line is the naïve $\Lambda \mathrm{CDM}$ (slope 3 ) prediction with no feedback, while the brown dashed line is the (slope 4) prediction from MOND. Note that the inclination of Ho II is uncertain (Gentile et al. 2012).

At $z=0$, it is possible that $\mathrm{SN}$ feedback at intermediate and low masses combines with entropy feedback from photoionization at low masses to conspire to give a linear baryonic Tully-Fisher relation (BTFR), as observed (see Fig. 15). This is an important issue as the normalization, slope and linearity of the BTFR have been used as evidence for MOdified Newtonian Dynamics (MOND, Milgrom, 1983) and against $\Lambda \mathrm{CDM}$. Indeed, McGaugh (2011) has pointed out that the observations of baryonic mass (stars plus cold gas) as a function of the velocity of the flat part of the rotation curve is very well matched by the MOND prediction (with no free parameters). He argues that considerable fine-tuning is required to bring the naïve $\Lambda \mathrm{CDM}$ (slope 3 ) prediction with no feedback to match the data. Our best-fit model matches the data equally well (with three free parameters), but the entropy feedback (photoionization) 
implies that the relation should curve at low masses, except if one considers galaxies in which the bulk of the stars formed before the reionization epoch $(z>6)$. Dutton (2012) also matched the BTFR data with a SAM. Moreover, two of the lowest mass galaxies in the McGaugh sample have rotation velocities corrected for asymmetric drift (see Begum \& Chengalur, 2004), after which the rotation curve of these galaxies is roughly linear with radius, and therefore depends on the last data point obtained with radioobservations, contradicting the flat part of the rotation curve sought by McGaugh.
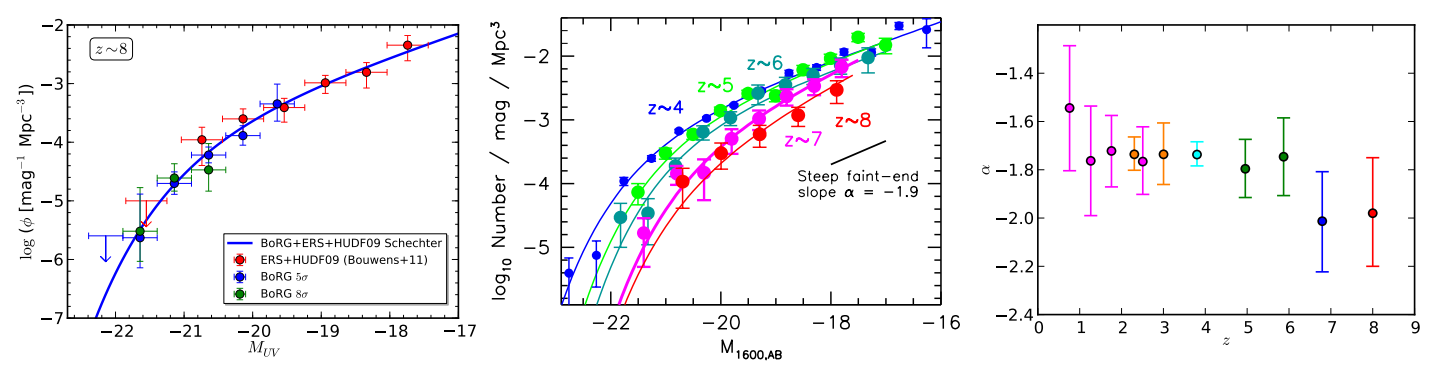

Fig. 16 Left: Galaxy luminosity function at $z=8$ (Bradley et al. 2012). Middle and right: evolution of the galaxy luminosity function (Bouwens et al., 2012) and of its faint-end slope (Bradley et al., 2012).

Intermediate-mass dwarfs are present at high redshift and have a steep luminosity function (Bradley et al., 2012, see Fig. 16,. They may contribute significantly to the reionization of the Universe.

\subsection{Gas accretion versus mergers}

Star formation seems to be too complex to be simply gravity-induced. Merging and AGN triggering are culprits for playing possible roles. What seems to be progressively clear is that there are two distinct modes of star formation. One mode occurs without any intervention from AGN and is characteristic of disk galaxies such as the MW, on a time-scale of order at least several galactic rotation times. Another mode is more intense, occurring on a relatively rapid time-scale, and involves the intervention of AGN, at least for quenching and possibly for enhancement or even triggering.

The most important aspect of star formation is the role of the raw material, cold gas. There are two modes of gas accretion, which may be classified as cold flows/minor mergers and major mergers/cooling flows. The former provide supplies of cold gas along filaments, the latter a source of hot gas which may cool and feed star formation.

The cold flows occur in filamentary streams that follow the cosmic web of large-scale structure (see Fig. 17), and include minor mergers via the dwarf galaxies that similarly trace the web (Dekel et al. 2009). Theory suggests that, at low redshift, gas accretion by cold streams is important, and that the cold streams are invariably clumpy and essentially indistinguishable from minor mergers of gas-rich dwarfs. Major galaxy mergers account for the observed morphological distortions that are more common at high $z$, and generally lead to cloud agglomeration, angular momentum loss and cooling flows that feed star formation (Bournaud et al., 2011b).

Observationally, one finds that cold flows are rarely if at all observed. This is presumably because of the small covering factor of the filaments (Stewart et al., 2011; Faucher-Giguère \& Kereš, 2011). Indirect evidence in favor of cold accretion comes from studies of star formation in dwarfs. The best example may be the Carina dwarf where three distinct episodes of star formation are found (Tolstoy et al. 2009). However at high redshift, major mergers between galaxies are common. Indeed, UltraLuminous Infrared Galaxies (ULIRGs), whose SFRs are huge, are invariably undergoing major, often multiple, gas-rich mergers (Borne et al. 2000) and dominate the cosmic SFR history at $z \gtrsim 2$, whereas 


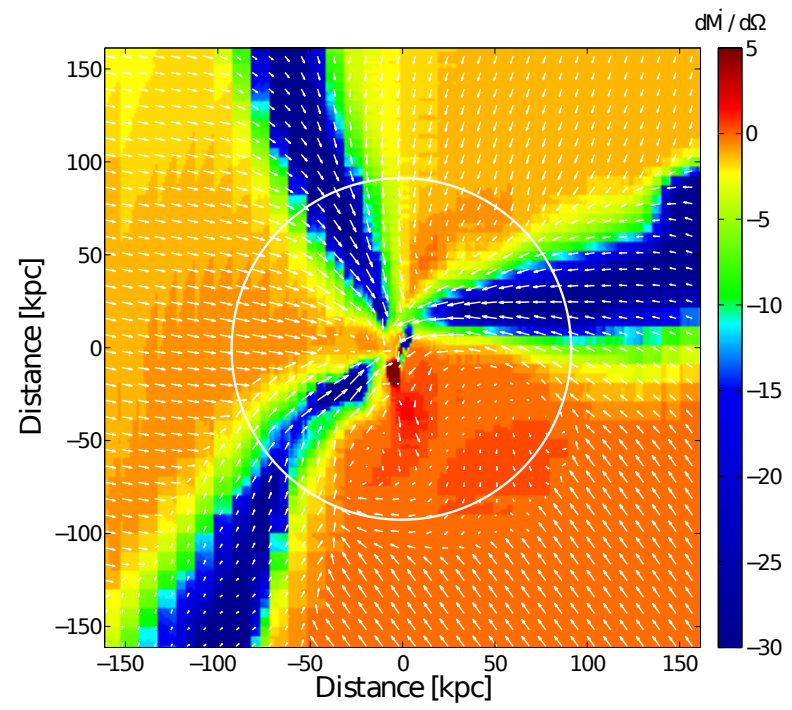

Fig. 17 Mass flux map of a $M_{v}=10^{12} \mathrm{M}_{\odot}$ halo at $z=2.5$ from a hydrodynamical simulation (Dekel et al. 2009). The circle denotes the virial radius.

normal star-forming galaxies predominate at low $(z \lesssim 2)$ redshift (Le Borgne et al., 2009). This certainly favors the idea of massive spheroid formation by major mergers.

Using, their analytical model of galaxy formation on top of a high-resolution cosmological simulation, Cattaneo et al. (2011) show that only in massive galaxies $\left(m_{\text {stars }}>10^{11} h^{-1} \mathrm{M}_{\odot}\right)$ do galaxy mergers contribute to the bulk of the stellar mass growth (see also Guo \& White, 2008, who analyzed a simulation with 11 times worse mass resolution) and these mergers are mainly 'dry' (gas-poor). As one goes to lower stellar masses (down to their simulation's effective resolution limit of $10^{10.6} h^{-1} \mathrm{M}_{\odot}$ ) the role of mergers sharply diminishes, suggesting, by extrapolation, that mergers are, in general, unimportant for the mass growth of both these intermediate-mass galaxies and low-mass galaxies, for which the bulk of the growth must be by gas accretion. Nevertheless, among those rare intermediate-mass galaxies built by mergers, the growth in mass is mostly in 'wet' (gas-rich) and minor mergers. In particular, the non-dominant cluster galaxies, known to be mostly dwarf ellipticals, are rarely built by mergers.

The sudden dominance of major mergers at high galaxy masses is confirmed by trends with stellar mass of the colors, color gradients and elongations of SDSS galaxies (Tremonti et al., 2004; Bernardi et al., 2010, see also van der Wel et al., 2009; Thomas et al., 2010). At lower masses (and low redshift), minor mergers are required to account for sizes and masses (McLure et al., 2012, López-Sanjuan et al. 2012).

Herschel observations of the Main Sequence of galaxy formation (SFR versus stellar mass) suggests that starbursts, commonly associated with major mergers, are displaced to higher mass and SFR, but only account for $10 \%$ of the SFR density at $z=2$ (Rodighiero et al. 2011). However, this conclusion depends critically on the $\sim 100$ Myr timescale assumed for the starbursts. If the starbursts had shorter duration, say $20 \mathrm{Myr}$, given their effective observation time of $\sim 1 \mathrm{Gyr}$, they would account for as much as $50 \%$ of the star formation at $z \sim 2$. It is difficult to gauge independent estimates of starburst age, but for example the UV continuum flattening observed at high $z$ for luminous star-forming galaxies favors a younger starburst age (González et al. 2011b) as would possible SED corrections for nebular emission. 


\subsection{Initial stellar mass function}

The IMF of stars forming in galaxies is usually treated as universal in galaxy formation modelling. There has been a recent flurry of papers finding evidence for a systematic steepening, from the Chabrier (2003) to Salpeter (1955) IMFs, in massive early type galaxies. From a spectral absorption line analysis, a correlation of IMF steepening with enhanced velocity dispersion, $[\mathrm{Mg} / \mathrm{Fe}]$ and sodium abundance is reported by (Conroy \& van Dokkum, 2012). A similar result is reported for stacked massive galaxy spectra (Ferreras et al. 2012). The modeling of the internal kinematics of early-type galaxies using in-

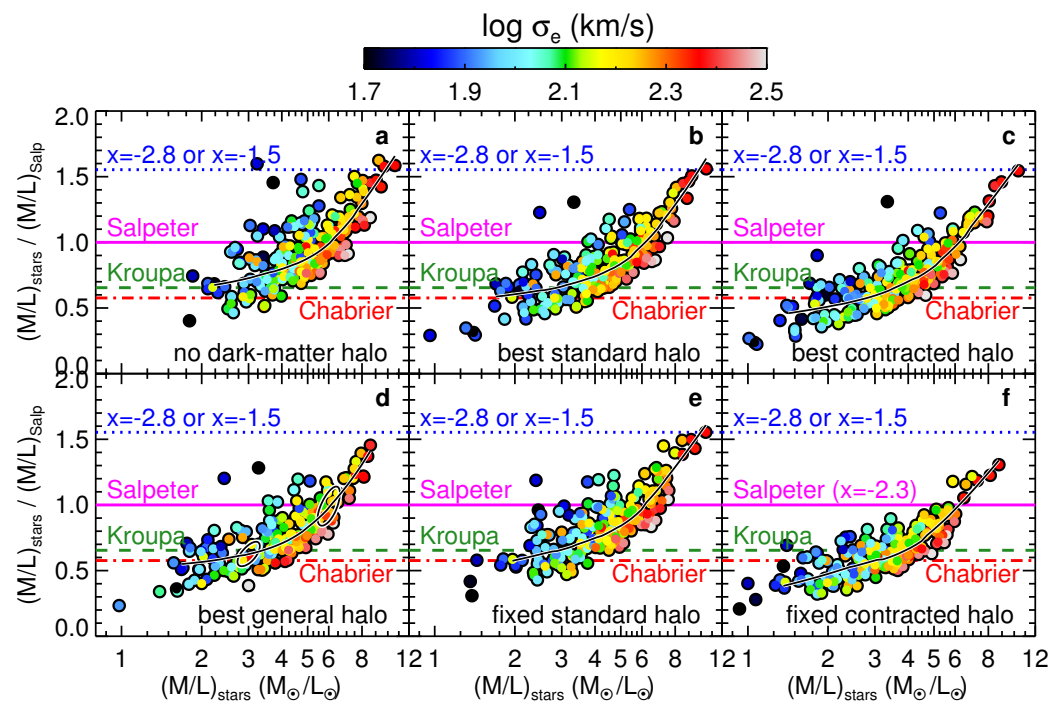

Fig. 18 Stellar mass-to-light ratio inferred from kinematical modeling (after subtracting off the contribution of the DM component), normalized to Salpeter ratio inferred from stellar populations versus stellar $M / L$ from kinematical modeling, for six DM models (Cappellari et al., 2012).

tegral field spectroscopy provides evidence for steeper IMFs (regardless of many plausible assumptions on the DM) in increasingly more massive galaxies (Cappellari et al. 2012, see Fig. 18). Lensing plus gas kinematics provides evidence for a Salpeter-like IMF in several massive ellipticals (Dutton et al. 2012b). There may also be a correlation of a steeper IMF with the densest massive galaxies (Dutton et al. 2012a). All of these studies report increasing $\mathrm{M} / \mathrm{L}$ with increasing spheroid velocity dispersion and $\alpha /[\mathrm{Fe}]$.

The possible degeneracy between IMF and DM fraction and shape is a concern because the DM profile steepens as a consequence of adiabatic contraction. While, Cappellari et al. (2012) tried a variety of DM models that do not significantly influence their result (since they only probed the region where dark matter accounts for at best $20 \%$ of the mass), only one study (Sonnenfeld et al., 2012) so far has cleanly broken the degeneracy with the dark matter profile: By using a double Einstein ring, Sonnenfeld et al. found a strong case for a Salpeter IMF. The adiabatic contraction of the DM is within the range found by Gnedin et al. (2004).

The implications of a steeper IMF in massive galaxies for galaxy formation models remain to be explored. The increased efficiency of star formation required at early epochs will certainly provide further tensions with the need to leave a substantial gas supply at late epochs for the observed late evolution observed for low mass galaxies, as discussed below. 


\subsection{Feedback and AGN}

Quenching of star formation has been largely motivated by the apparent success of SMBH feedback in reproducing the scaling and normalization of the black hole mass-spheroid velocity dispersion $M_{\mathrm{BH}}-$ $\sigma_{v}$ ) relation, as first proposed by Silk \& Rees (1998). SAMs indeed demonstrate that AGN feedback is able to quench star formation in massive elliptical galaxies (Croton et al., 2006; Bower et al., 2006, Cattaneo et al. 2006; Somerville et al., 2008). One can reproduce the fairly sharp cut-off in the bright end of the galaxy luminosity function (Bell et al., 2003, Panter et al., 2007). These SAMs do not require "quasar mode" AGN feedback with Eddington luminosities.
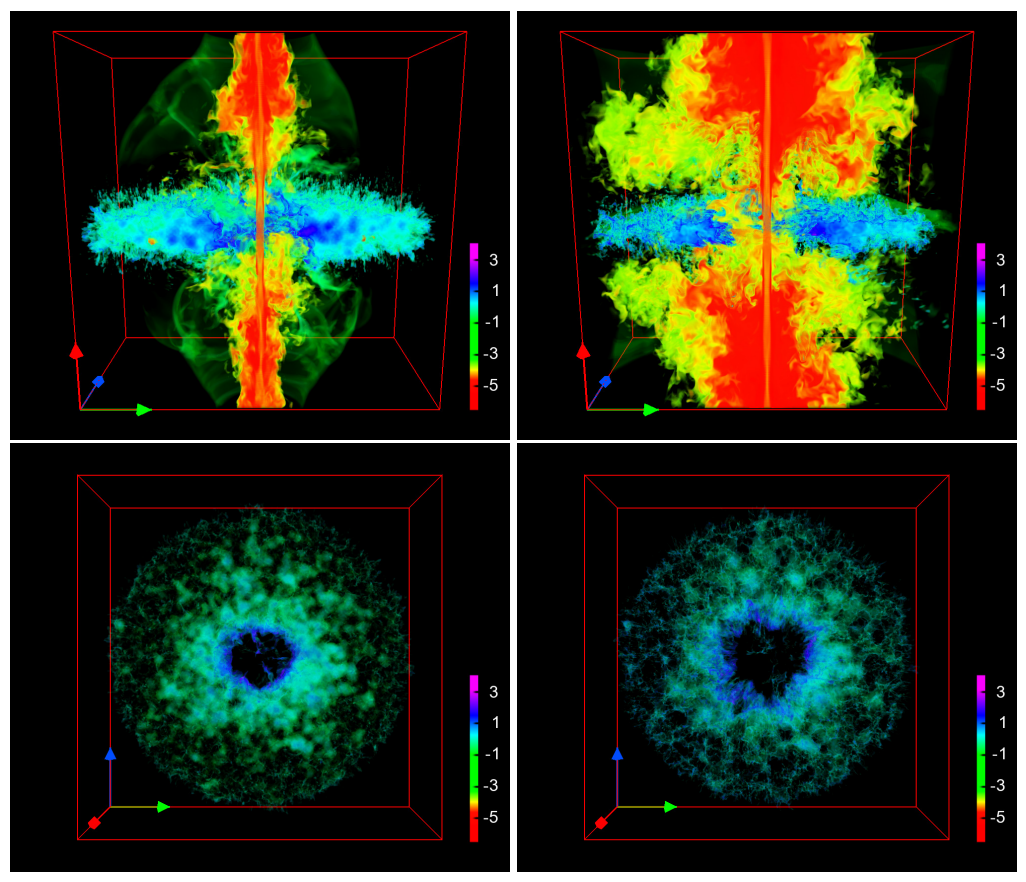

Fig. 19 Simulations (in $32 \mathrm{kpc}$ box) of AGN feedback at 14 (left) and 22 Myr (right) after the onset of the jet, in edge-on (top) and face-on (bottom) views of log density (Gaibler et al. 2011)

High resolution hydrodynamical cosmological simulations indeed show that while cold streams initially feed the black hole, transferring angular momentum to produce central disks (Dubois et al. 2012a) that become gravitationally unstable and feed the compact bulge through migration of clumps (see Bournaud et al. 2011a), the cold flows are eventually interrupted by AGN-driven super-winds (Dubois et al. 2012b).

However the physics of driving SMBH outflows is still not well understood. One issue is that momentum-driven winds fail to account for the normalization of the $M_{\mathrm{BH}}-\sigma_{v}$ relation (Silk \& Nusser. 2010; Debuhr et al. 2012), with the shortfall being about a factor of 10. This momentum deficit can be supplied by radio jet-driven outflows (Wagner \& Bicknell, 2011), which also account for the observed high velocities of entrained cold gas (Wagner et al. 2012). Alternative or complementary possibilities, possibly more relevant to radio-quiet quasars, include positive feedback from outflow-triggered star formation (Silk \& Norman, 2009, Gaibler et al., 2011, see Fig. 19) and energy-driven outflows (FaucherGiguere \& Quataert, 2012). Nearby AGN show dense molecular rings surrounding circumnuclear rings 
of star formation (Sani et al., 2012), reminiscent of the simulated triggering of star formation (Gaibler et al., 2011).

SMBHs are generally found to correlate with bulges rather than with disks, pseudobulges or dark halos (Ho, 2007; Kormendy et al., 2011, Kormendy \& Bender, 2011), although disk galaxies appear to follow a similar $M_{\mathrm{BH}}-\sigma_{v}$ relation, albeit with more scatter (Graham et al., 2011). This would simplify formation mechanisms, suggesting that bulges and SMBH grow together, perhaps self-regulating each other. Massive black hole growth at early epochs seems to be (just) achievable by gas accretion. Large cosmological simulations (Di Matteo et al., 2012, see also Li et al., 2007; Sijacki et al., 2009; Khandai et al. 2012) have shown that primordial massive BHs can grow by cold filamentary infall, and acquire masses of up to several billion solar masses by $z=6$ in the most massive halos $\left(M_{\mathrm{vir}} \simeq 10^{12-13} \mathrm{M}_{\odot}\right)$.

Insight into black hole growth is provided by looking for extreme deviations in the $M_{\mathrm{BH}}-\sigma_{v}$ relation. Massive black holes seem to be in place at high redshift before spheroids (Wang et al., 2011). This is also the case for a nearby starbust galaxy containing an AGN but without any matching spheroid or indeed massive stellar component (Reines \& Deller, 2012). On the other hand, SMGs seem to contain relatively low mass black holes for their stellar content (Alexander et al., 2008).

\section{FUTURE PROSPECTS IN OBSERVATIONS}

A clue as to the nature of a possible solution may come from the fact that quasars also reveal luminosity downsizing. This translates into downsizing of central SMBH mass. One might be able to connect the two phenomena if feedback from AGN was initially positive and also a strongly nonlinear function of SMBH mass. Predictions of positive feedback include circumnuclear rings on 10-100 pc scales in starforming AGN. These should be resolvable with ALMA, via both molecular lines that probe pressurized molecular gas and FIR fine-structure lines that probe the interplay of intense FUV radiation fields with photodissociation regions (PDRs and XDRs).

More conventionally, the evidence for AGN quenching of star formation seems strong. Superwinds driven by AGN are capable of depleting the reservoir of star-forming gas over relatively short timescales. However, questions remain as to the relative roles of AGN winds, jet-driven bubbles, SNe, and radiation pressure especially from OB star clusters. No doubt, JWST, as well as 30+meter telescopes such as ELT will complement HST by producing spectacular IR images of star formation, AGN and outflows at work. Accretion of neutral gas will be studied at high sensitivity by SKA. Ultimately one needs a spectroscopic survey akin to SDSS at $z=1-2$ and this will be provided by the Subaru Prime Focus Spectrograph with optical and NIR capability. The next decade should bring a vast increase in our phenomenological understanding of the basic processes at play in galaxy formation and evolution.

\section{FUTURE PROSPECTS IN ASTROPHYSICAL THEORY}

Theory lacks adequate resolution and physics. Of course these issues are intricately connected. One needs to tackle baryon physics and the associated possibilities for feedback. Today, state-of-the-art cosmological simulations of the MW with gas and star formation, such as the ERIS simulation (Guedes et al., 2011), provide only $\approx 100 \mathrm{pc}$ resolution. Hence, in current simulations, the gas and star formation physics is included in an ad hoc way, because of the resolution limitation. For example, while stars are known to form in the dense cores — of density $\gtrsim 10^{5} \mathrm{~cm}^{-3}$ — of Giant Molecular Clouds, the current hydrodynamical simulations adopt SF thresholds of typically $1 \mathrm{~cm}^{-3}$ and always $\lesssim 10^{2} \mathrm{~cm}^{-3}$. Sharp

increases of the SF density threshold result in moving the SF regions outside of the nucleus (Teyssier et al. 2010). However, in reality, it is the unresolved subgrid physics that determines the actual threshold, if one even exists. Mastery of the required subparsec-scale physics will take time, but there is no obvious reason why we cannot achieve this goal with orders of magnitude improvement in computing power.

For the moment, phenomenology drives all modelling. This is true especially for local star formation. A serious consequence is that physics honed on local star-forming regions, where one has high 
resolution probes of star-forming clouds and of ongoing feedback, may not necessarily apply in the more extreme conditions of the early universe.

One issue that arises frequently is whether the perceived challenges to $\Lambda \mathrm{CDM}$ justify a new theory of gravity. From MOND (Milgrom 1983) onwards, there are any number of alternative theories that are designed to explain certain observations. However, none can explain the ensemble of observations any better than $\Lambda \mathrm{CDM}$, nor do they rely on solid physical grounds. But to the extent that any unexplained anomalies exist, these are invariably at no more than the $2 \sigma$ level of significance. It seems that such "evidence" is not adequate motivation for abandoning Einstein-Newton gravity. Indeed, while it is overwhelmingly clear that there are many potential discrepancies with $\Lambda \mathrm{CDM}$, we have certainly not developed the optimal $\Lambda \mathrm{CDM}$ theory of galaxy formation: the current models do not adequately include the baryons nor do we reliably understand star formation, let alone feedback. Other MOND-related issues are reviewed in Famaey \& McGaugh (2011), including challenges raised by the apparent emptiness of local voids and satellite phase space correlations. However, we regard these as more a matter of absorbing the significance of ever deeper galaxy and $21 \mathrm{~cm}$ surveys, on the one hand (for example, deep blind HI surveys show that gas-rich galaxies are the least clustered of any galaxy population Martin et al. 2012), and on the other hand, of questioning the details of hitherto inadequately modelled baryonic physics, as developed for example in Zolotov et al. (2012). Whether appeal to alternative gravity is justified by inadequate baryonic physics is a question of judgement at this point. Here is a summary of many of these failures: we cite some key reasons why $\Lambda$ CDM does not yet provide a robust explanation of the observations: we list below several examples that represent challenges for theorists.

1. Massive bulgeless galaxies with thin disks are reasonably common (Kormendy et al., 2010). Simulations invariably make thick disks and bulges. Indeed, the bulges are typically overly massive relative to the disks for all galaxies other than S0s. Massive thin disks are especially hard to simulate unless very fine-tuned feedback is applied. A consensus is that the feedback prescriptions are far from unique (Scannapieco et al. 2012). One appealing solution involves SN feedback. This drives a galactic fountain that feeds the bulge. A wind is driven from the bulge where star formation is largely suppressed for sufficiently high feedback (Brook et al. 2012). Another proposal includes radiation pressure from massive stars as well as $\mathrm{SNe}$. The combined feedback helps expand the halo expansion, thereby limiting dynamical friction and bulge formation (Macciò et al. 2012).

2. Dark matter cores are generally inferred in dwarf spheroidal galaxies, whereas $\Lambda \mathrm{CDM}$ theory predicts a cusp, the NFW profile. Strong SN feedback can eject enough baryons from the innermost region to create a core (Governato et al., 2010; Pontzen \& Governato, 2012), but this requires high early SN feedback or a series of implausibly short bursts of star formation.

3. The excessive predicted numbers of dwarf galaxies are one of the most cited problems with $\Lambda$ CDM. The discrepancy amounts to two orders of magnitude. The issue of dwarf visibility is addressed by feedback that ejects most of the baryons and thereby renders the dwarfs invisible, at least in the optical bands. There are three commonly discussed mechanisms for dwarf galaxy feedback: reionization of the universe at early epochs, $\mathrm{SNe}$, and (ram pressure and tidal) stripping. AGNdriven outflows via intermediate mass black holes provide another alternative to which relatively little attention has been paid (Silk \& Nusser. 2010).

None of these have so far been demonstrated to provide definitive solutions. Reionization only works for the lowest mass dwarfs. The ultrafaint dwarfs in the MW may be fossils of these first galaxies (as checked by detailed models Koposov et al. 2009, Salvadori \& Ferrara, 2009, Bovill \& Ricotti, 2011). It is argued that SN feedback solves the problem for the more massive dwarfs (Macciò et al., 2010). However, this conclusion is disputed by Boylan-Kolchin et al. (2011), who use the Aquarius simulations (Springel et al. 2008) to predict more massive dwarfs in dark-matteronly simulations than are observed. These authors argue that the relatively massive dwarfs should form stars, and we see no counterparts of these systems, apart possibly from rare massive dwarfs such as the Magellanic Clouds. We have previously remarked that omission of baryonic physics biases the dark matter-only simulations to an overstatement of the problem by overpredicting dwarf central densities (Zolotov et al., 2012). 
4. The SFE in dwarfs is highly debated. Let us put aside the high SFE at early epochs that is required to obtain strong feedback in order to generate cores. For example, it is possible that intermediate mass black holes could be invoked to solve this problem and simultaneously generate the required low baryon fraction (Peirani et al., 2012).

In order to obtain the required late epoch evolution (Weinmann et al., 2012), one might appeal to a lower SFE in dwarfs, plausibly associated with low metallicities and hence low dust and $\mathrm{H}_{2}$ content. Models based on metallicity-regulated star formation can account for the numbers and radial distribution of the dwarfs by a decreasing SFE (Kravtsov, 2010). This explanation is disputed by Boylan-Kolchin et al. (2011), who infer a range in SFEs for the dwarfs of some two orders of magnitude. A similar result appeals to varying the halo mass threshold below which star formation must be suppressed to account for the dwarf luminosity function, whereas the stellar masses of many observed dwarfs violate this condition (Ferrero et al., 2011). Finally, tidal stripping may provide a solution (Nickerson et al. 2011), at least for the inner dwarfs.

5. Another long-standing problem relates to downsizing. Massive galaxies are in place before lower mass galaxies as measured by stellar mass assembly, and their star formation time-scales and chemical evolution time-scales at their formation/assembly epoch are shorter. One popular explanation (Cattaneo et al. 2008) is that galaxies cannot accrete/retain cold gas in massive halos, either because of AGN feedback or because of virial shocks that prevent the gas supply of the disk in cold filaments (Birnboim \& Dekel, 2003).

6. It is possible to develop galaxy formation models with suitable degrees and modes of feedback that address many of these issues. However, a major difficulty confronted by all SAMs is that the evolution of the galaxy luminosity function contradicts the data, either at high or at low redshift. The SAMs that are normalized to low redshift and tuned to account for the properties of local galaxies fail at high redshift by generating too many red galaxies (Fontanot et al. 2009). Too few blue galaxies are predicted at $z=0.3$. This problem has been addressed by including AGB stars in the stellar populations. This fix results in a more rapid reddening time-scale by speeding up the evolution of the rest-frame near-infrared galaxy luminosity function (Henriques et al., 2011). There is a price to be paid however: now there are excess numbers of blue galaxies predicted at $z=0.5$.

7. There is a well-known difficulty in matching both the galaxy luminosity function and Tully-Fisher scaling relation, even at $z=0$. Reconciliation of the Tully-Fisher zero point with the galaxy luminosity function requires too high an efficiency of star formation (Guo et al., 2010). In fact, the problem is even worse: the models of massive spirals tuned to fit the Tully-Fisher relation are too concentrated (McCarthy et al. 2012). This is a reflection of the over-massive bulge problem in disk galaxies that simply refuses to go away (Navarro \& Steinmetz, 2000; Abadi et al. 2003).

8. The luminosity function problem is most likely related to another unexplained property of high redshift galaxies. The SSFR evolution at high $z$ is very different from that at low $z$. Essentially, it saturates. One finds an infrared Main Sequence of galactic SFRs: SFR versus $M_{*}$ (Elbaz et al. 2011). Neither the slope nor the scatter are adequately understood. Starburst galaxies lie above the Main Sequence, but the fraction of cosmic star formation in these systems depends on inadequately justified assumptions about starburst duration. For example, nebular emission and dust extinction affect infrerred ages, and one cannot easily understand the blue continuum slopes oberved at high redshift and lower UV luminosities (Bouwens et al., 2011).

9. The observed rapid growth of early-type galaxy sizes since $z=2$ for fixed stellar mass cannot be reproduced in SAMs or analytical models (Cimatti et al., 2012): at $z=2$ galaxies are too compact.

10. Much has been made of nearby rotation curve wiggles that trace similar dips in the stellar surface density that seemingly reduce the significance of any dark matter contribution. Maximum disks optimize the contribution of stars to the rotation curve, and these wiggles are most likely associated with spiral density waves. A similar result may be true for low surface brightness gas-rich dwarf galaxies (Swaters et al. 2011).

11. High mass-to-light ratios are sometimes required for maximum disk models of spiral galaxy rotation curves, but these are easily accommodated if the IMF is somewhat bottom-heavy. The case for IMF variations has been made for several data sets, primarily for early-type galaxies (e.g., see van 
Dokkum \& Conroy, 2011). The LSB dwarfs are plausible relics of the building blocks expected in hierarchical formation theories.

12. Spiral arms are seen in the HI distribution in the outer regions of some disks. This tells us that significant angular momentum transfer is helping feed the optical inner disk. The baryon self-gravity is large enough that one does not for example need to appeal to a flattened halo, which might otherwise be problematic for the DM model (Bertin \& Amorisco, 2010).

13. The slope and normalization of the baryon Tully-Fisher relation do not agree with the simplest $\Lambda$ CDM prediction. The observed slope is approximately 4, similar to what is found for MOND (Milgrom, 1983), whereas $\Lambda$ CDM (without feedback) gives a slope of 3 (McGaugh, 2011, 2012), but fails to account for the observed dispersion and curvature.

14. The baryon fraction in galaxies is some $50 \%$ of the primordial value predicted by light element nucleosynthesis. These baryons are not in hot gaseous halos (Anderson \& Bregman, 2010). Convergence to the universal value on cluster scales is controversial: convergence to the WMAP value is seen for X-ray clusters above a temperature of $5 \mathrm{keV}$ (Dai et al., 2010), but could be as large as 30\% even for massive clusters (Andreon, 2010; Scannapieco et al., 2012). If the latter discrepancy were to be confirmed, one would need significant bias of baryons relative to dark matter, presumably due to feedback, on unprecedentedly large scales.

15. The distribution of the MW satellite galaxies in a great circle (Lynden-Bell, 1982) is unexpected in the $\Lambda$ CDM context (Kroupa et al. 2005). However, infall onto halos is not spherically symmetric (Aubert et al., 2004), and subhalos tend to lie in a plane (Libeskind et al., 2005). The details of the thickness of this plane remained to be settled (e.g., Kroupa et al. |2010 versus Libeskind et al. 2011).

16. There is a significant lack of galaxies in comparison with standard expectations in the Local Void close to the Local Group (Peebles, 2007, Tikhonov \& Klypin, 2009). But it is not yet clear whether this region fairly low galactic latitude region has been surveyed as closely as other regions.

17. Bulk flows are found over $100 \mathrm{Mpc}$ scales that are about two standard deviations larger than expected in $\Lambda \mathrm{CDM}$ (Feldman et al. 2010 ). The technique primarily uses Tully-Fisher and Fundamental Plane galaxy calibrators of the distance scale. An X-ray approach, calibrating via the kinetic Sunyaev \& Zeldovich (1972) effect (kSZE), claims the existence of a bulk flow out to 800 Mpc (Kashlinsky et al. 2010). However the discrepancies with $\Lambda$ CDM are controversial because of possible systematics. A recent detection of kSZE confirms pairwise bulk flows of clusters at $4 \sigma$ and is consistent with $\Lambda \mathrm{CDM}$ (Hand et al. 2012).

Several of these issues may be linked. For example, the analysis of Cappellari et al. (2012) that the IMF is non-universal, with shallower (top-heavy) IMFs for galaxies of lower velocity dispersion, can be linked with the known relations between velocity dispersion and metallicity (e.g., Allanson et al., 2009) to produce a relation between IMF and metallicity, which goes in the right direction: low-metallicity systems have top-heavy IMFs. Until now, observers assumed a universal IMF when deriving stellar masses. They have therefore overestimated the stellar masses of low-metallicity systems. We would like to think that this overestimation of $M_{*}$ might explain at the same time the evolution of the cosmic SSFR and that of galaxy sizes. Indeed, at high redshift, galaxies are expected to be more metal-poor, and the overestimate of their typical stellar masses will lead to an underestimate of their SSFRs, relative to those of lower-redshift galaxies. Therefore, the cosmic SSFR may not saturate at high redshift, which will make it easier to fit to models. At the same time, if high redshift galaxies have lower stellar masses than inferred from a universal IMF, then for a given stellar mass, they have larger sizes than inferred, and the too rapid evolution of galaxy sizes (relative to models) might disappear. We propose that observers replace stellar mass by $K$-band rest-frame luminosity, which, if properly measured, can serve as a useful proxy for stellar mass, independently of any assumed IMF.

In summary, it is clear that many problems await refinements in theoretical understanding. No doubt, these will come about eventually as numerical simulations of galaxy formation are refined to tackle subparsec scales. 
We are grateful to A. Cattaneo, B. Famaey, A. Graham, J. Kormendy, P. Kroupa, S. McGaugh, A. Pontzen and A. Tutukov for very useful comments.

\section{References}

Abadi M. G., Navarro J. F., Steinmetz M., Eke V. R., 2003, ApJ, 591, 499

Agertz O., Teyssier R., Moore B., 2011, MNRAS, 410, 1391

Alexander D. M., Brandt W. N., Smail I., et al., 2008, AJ, 135, 1968

Allanson S. P., Hudson M. J., Smith R. J., Lucey J. R., 2009, ApJ, 702, 1275

Anderson M. E., Bregman J. N., 2010, ApJ, 714, 320

Andreon S., 2010, MNRAS, 407, 263

Aubert D., Pichon C., Colombi S., 2004, MNRAS, 352, 376

Baldry I. K., Glazebrook K., Brinkmann J., et al., 2004, ApJ, 600, 681

Begum A., Chengalur J. N., 2004, A\&A, 413, 525

Behroozi P. S., Conroy C., Wechsler R. H., 2010, ApJ, 717, 379

Behroozi P. S., Wechsler R. H., Conroy C., 2012, ApJ, submitted, arXiv:1207.6105

Bell E. F., Baugh C. M., Cole S., Frenk C. S., Lacey C. G., 2003, MNRAS, 343, 367

Bender R., Burstein D., Faber S. M., 1992, ApJ, 399, 462

Benson A. J., 2012, New Ast, 17, 175

Berlind A. A., Weinberg D. H., 2002, ApJ, 575, 587

Bernardi M., Shankar F., Hyde J. B., Mei S., Marulli F., Sheth R. K., 2010, MNRAS, 404, 2087

Bertin G., Amorisco N. C., 2010, A\&A, 512, A17

Bigiel F., Leroy A., Walter F., 2011, in IAU Symposium, Vol. 270, Computational Star Formation, Alves J., Elmegreen B. G., Girart J. M., Trimble V., eds., pp. 327-334

Birnboim Y., Dekel A., 2003, MNRAS, 345, 349

Blanchard A., Valls-Gabaud D., Mamon G. A., 1992, A\&A, 264, 365

Boomsma R., Oosterloo T. A., Fraternali F., van der Hulst J. M., Sancisi R., 2008, A\&A, 490, 555

Borne K. D., Bushouse H., Lucas R. A., Colina L., 2000, ApJ, 529, L77

Bouché N., Dekel A., Genzel R., et al., 2010, ApJ, 718, 1001

Bournaud F., Dekel A., Teyssier R., Cacciato M., Daddi E., Juneau S., Shankar F., 2011a, ApJ, 741, L33

Bournaud F., Powell L. C., Chapon D., Teyssier R., 2011b, in IAU Symposium, Vol. 271, IAU Symposium, Brummell N. H., Brun A. S., Miesch M. S., Ponty Y., eds., pp. 160-169

Bouwens R. J., Illingworth G. D., Oesch P. A., et al., 2011, ApJ, in press, arXiv:1109.0994

Bouwens R. J., Illingworth G. D., Oesch P. A., et al., 2012, ApJ, 752, L5

Bovill M. S., Ricotti M., 2011, ApJ, 741, 18

Bower R. G., 1991, MNRAS, 248, 332

Bower R. G., Benson A. J., Malbon R., et al., 2006, MNRAS, 370, 645

Boylan-Kolchin M., Bullock J. S., Kaplinghat M., 2011, MNRAS, 415, L40

-, 2012, MNRAS, 422, 1203

Boylan-Kolchin M., Springel V., White S. D. M., Jenkins A., Lemson G., 2009, MNRAS, 398, 1150

Bradley L. D., Trenti M., Oesch P. A., et al., 2012, ApJ, submitted, arXiv:1204.3641

Brook C. B., Stinson G., Gibson B. K., Roškar R., Wadsley J., Quinn T., 2012, MNRAS, 419, 771

Cappellari M., McDermid R. M., Alatalo K., et al., 2012, Nature, 484, 485

Cattaneo A., Dekel A., Devriendt J., Guiderdoni B., Blaizot J., 2006, MNRAS, 370, 1651

Cattaneo A., Dekel A., Faber S. M., Guiderdoni B., 2008, MNRAS, 389, 567

Cattaneo A., Faber S. M., Binney J., et al., 2009, Nature, 460, 213

Cattaneo A., Mamon G. A., Warnick K., Knebe A., 2011, A\&A, 533, A5

Ceverino D., Dekel A., Bournaud F., 2010, MNRAS, 404, 2151

Chabrier G., 2003, PASP, 115, 763

Cimatti A., Nipoti C., Cassata P., 2012, MNRAS, 422, L62

Conroy C., van Dokkum P., 2012, ApJ, submitted, arXiv:1205.6473

Conroy C., Wechsler R. H., Kravtsov A. V., 2006, ApJ, 647, 201

Cooper A. P., Cole S., Frenk C. S., et al., 2010, MNRAS, 406, 744

Courtin J., Rasera Y., Alimi J.-M., Corasaniti P.-S., Boucher V., Füzfa A., 2011, MNRAS, 410, 1911

Crocce M., Fosalba P., Castander F. J., Gaztañaga E., 2010, MNRAS, 403, 1353

Croton D. J., Springel V., White S. D. M., et al., 2006, MNRAS, 365, 11

Dai X., Bregman J. N., Kochanek C. S., Rasia E., 2010, ApJ, 719, 119

De Lucia G., Blaizot J., 2007, MNRAS, 375, 2

de Vaucouleurs G., 1961, ApJS, 5, 233

Debuhr J., Quataert E., Ma C.-P., 2012, MNRAS, 420, 2221

Dekel A., Birnboim Y., Engel G., et al., 2009, Nature, 457, 451

Dekel A., Silk J., 1986, ApJ, 303, 39

Di Matteo T., Khandai N., DeGraf C., Feng Y., Croft R. A. C., Lopez J., Springel V., 2012, ApJ, 745, L29

D’Onghia E., Besla G., Cox T. J., Hernquist L., 2009, Nature, 460, 605 
Dubois Y., Devriendt J., Slyz A., Teyssier R., 2012a, MNRAS, 420, 2662

Dubois Y., Pichon C., Devriendt J., Silk J., Haehnelt M., Kimm T., Slyz A., 2012b, MNRAS, submitted, arXiv: 1206.5838

Dutton A. A., 2012, MNRAS, in press, arXiv:1206.1855

Dutton A. A., Mendel J. T., Simard L., 2012a, MNRAS, 422, L33

Dutton A. A., Treu T., Brewer B. J., Marshall P. J., Auger M. W., Barnabe M., Koo D. C., Bolton A. S., Koopmans L. V. E., 2012b, MNRAS, submitted, arXiv: 1206.4310

Elbaz D., Dickinson M., Hwang H. S., et al., 2011, A\&A, 533, A119

Elmegreen B. G., 1997, in Revista Mexicana de Astronomia y Astrofisica, vol. 27, Vol. 6, Revista Mexicana de Astronomia y Astrofisica Conference Series, Franco J., Terlevich R., Serrano A., eds., p. 165

Evrard A. E., 1988, MNRAS, 235, 911

Faber S. M., 1973, ApJ, 179, 731

Faber S. M., Jackson R. E., 1976, ApJ, 204, 668

Famaey, B., McGaugh, S., 2011, Living Reviews in Relativity, in press, arXiv:1112.3960

Fanidakis N., Baugh C. M., Benson A. J., Bower R. G., Cole S., Done C., Frenk C. S., 2011, MNRAS, 410, 53

Faucher-Giguère C.-A., Kereš D., 2011, MNRAS, 412, L118

Faucher-Giguere C.-A., Quataert E., 2012, MNRAS, in press, arXiv:1204.2547

Feldman H. A., Watkins R., Hudson M. J., 2010, MNRAS, 407, 2328

Ferrarese L., Merritt D., 2000, ApJ, 539, L9

Ferreras I., La Barbera F., de Carvalho R. R., de la Rosa I. G., Vazdekis A., Falcon-Barroso J., Ricciardelli E., 2012, ApJ, submitted, arXiv:1206.1594

Ferrero I., Abadi M. G., Navarro J. F., Sales L. V., Gurovich S., 2011, MNRAS, submitted, arXiv:1111.6609

Fontanot F., De Lucia G., Monaco P., Somerville R. S., Santini P., 2009, MNRAS, 397, 1776

Frenk C. S., White S. D. M., Bode P., et al., 1999, ApJ, 525, 554

Gaibler V., Khochfar S., Krause M., Silk J., 2011, MNRAS, in press, arXiv:1111.4478

Gentile G., Angus G. W., Famaey B., Oh S.-H., de Blok W. J. G., 2012, A\&A, 543, A47

Genzel R., Tacconi L. J., Gracia-Carpio J., et al., 2010, MNRAS, 407, 2091

Gingold R. A., Monaghan J. J., 1977, MNRAS, 181, 375

Gnat O., Sternberg A., 2007, ApJS, 168, 213

Gnedin N. Y., 2000, ApJ, 542, 535

Gnedin O. Y., Kravtsov A. V., Klypin A. A., Nagai D., 2004, ApJ, 616, 16

González V., Bouwens R., Labbé I., Illingworth G., Oesch P., Franx M., Magee D., 2011b, ApJ, submitted, arXiv: 1110.6441

González V., Labbé I., Bouwens R. J., Illingworth G., Franx M., Kriek M., 2011a, ApJ, 735, L34

Governato F., Brook C., Mayer L., et al., 2010, Nature, 463, 203

Governato F., Zolotov A., Pontzen A., et al., 2012, MNRAS, 422, 1231

Graham A. W., Onken C. A., Athanassoula E., Combes F., 2011, MNRAS, 412, 2211

Grillmair C. J., 2009, ApJ, 693, 1118

Guedes J., Callegari S., Madau P., Mayer L., 2011, ApJ, 742, 76

Gunn J. E., Gott J. R., 1972, ApJ, 176, 1

Guo Q., White S., Boylan-Kolchin M., De Lucia G., Kauffmann G., Lemson G., Li C., Springel V., Weinmann S., 2011, MNRAS, 413, 101

Guo Q., White S., Li C., Boylan-Kolchin M., 2010, MNRAS, 404, 1111

Guo Q., White S. D. M., 2008, MNRAS, 384, 2

Gurovich S., Freeman K., Jerjen H., Staveley-Smith L., Puerari I., 2010, AJ, 140, 663

Hall M., Courteau S., Dutton A. A., McDonald M., Zhu Y., 2011, MNRAS, submitted, arXiv:1111.5009

Hand N., Addison G. E., Aubourg E., et al., 2012, Phys. Rev. Lett., 109, 041101

Heald G., Józsa G., Serra P., Zschaechner L., Rand R., Fraternali F., Oosterloo T., Walterbos R., Jütte E., Gentile G., 2011, A\&A, 526, A118

Henriques B., Maraston C., Monaco P., Fontanot F., Menci N., De Lucia G., Tonini C., 2011, MNRAS, 415, 3571

Ho L. C., 2007, ApJ, 668, 94

Jiang C. Y., Jing Y. P., Faltenbacher A., Lin W. P., Li C., 2008, ApJ, 675, 1095

Kashlinsky A., Atrio-Barandela F., Ebeling H., Edge A., Kocevski D., 2010, ApJ, 712, L81

Kaviraj S., Peirani S., Khochfar S., Silk J., Kay S., 2009, MNRAS, 394, 1713

Kennicutt Jr. R. C., Calzetti D., Walter F., et al., 2007, ApJ, 671, 333

Khandai N., Feng Y., DeGraf C., Di Matteo T., Croft R. A. C., 2012, MNRAS, 423, 2397

Khochfar S., Silk J., 2011, MNRAS, 410, L42

Knebe A., Knollmann S. R., Muldrew S. I., et al., 2011, MNRAS, 415, 2293

Koposov S. E., Yoo J., Rix H.-W., Weinberg D. H., Macciò A. V., Escudé J. M., 2009, ApJ, 696, 2179

Kormendy J., Bender R., 2011, Nature, 469, 377

,- 2012, ApJS, 198, 2

Kormendy J., Bender R., Cornell M. E., 2011, Nature, 469, 374

Kormendy J., Drory N., Bender R., Cornell M. E., 2010, ApJ, 723, 54

Kravtsov A., 2010, Advances in Astronomy, 2010, 281913 
Kravtsov A. V., Klypin A. A., Khokhlov A. M., 1997, ApJS, 111, 73

Kroupa P., Famaey B., de Boer K. S., et al., 2010, A\&A, 523, A32

Kroupa P., Theis C., Boily C. M., 2005, A\&A, 431, 517

Krumholz M. R., Dekel A., 2012, ApJ, 753, 16

Krumholz M. R., Dekel A., McKee C. F., 2012, ApJ, 745, 69

Lacey C., Cole S., 1993, MNRAS, 262, 627

Le Borgne D., Elbaz D., Ocvirk P., Pichon C., 2009, A\&A, 504, 727

Li C., White S. D. M., 2009, MNRAS, 398, 2177

Li Y., Hernquist L., Robertson B., Cox T. J., Hopkins P. F., Springel V., Gao L., Di Matteo T., Zentner A. R., Jenkins A., Yoshida N., 2007, ApJ, 665, 187

Libeskind N. I., Frenk C. S., Cole S., Helly J. C., Jenkins A., Navarro J. F., Power C., 2005, MNRAS, 363 , 146

Libeskind N. I., Knebe A., Hoffman Y., Gottlöber S., Yepes G., Steinmetz M., 2011, MNRAS, 411, 1525

López-Sanjuan C., Le Fèvre O., Ilbert O., et al., 2012, A\&A, submitted, arXiv:1202.4674

Lynden-Bell D., 1982, The Observatory, 102, 202

Macciò A. V., Kang X., Fontanot F., Somerville R. S., Koposov S., Monaco P., 2010, MNRAS, 402, 1995

Macciò A. V., Stinson G., Brook C. B., Wadsley J., Couchman H. M. P., Shen S., Gibson B. K., Quinn T., 2012, ApJ, 744, L9

Magorrian J., Tremaine S., Richstone D., et al., 1998, AJ, 115, 2285

Mamon G. A., Tweed D., Thuan T. X., Cattaneo A., 2012, in Dwarf Galaxies: Keys to Galaxy Formation and Evolution, Papaderos P., Recchi S., Hensler G., eds., Springer Verlag, Berlin, Heidelberg, pp. 39-46, arXiv: 1103.5349

Marchesini D., van Dokkum P. G., Förster Schreiber N. M., Franx M., Labbé I., Wuyts S., 2009, ApJ, 701, 1765

Marinoni C., Hudson M. J., 2002, ApJ, 569, 101

Marinoni C., Hudson M. J., Giuricin G., 2002, ApJ, 569, 91

Martin, A. M., Giovanelli, R., Haynes, M. P., Guzzo, L., 2012, ApJ, 750, 38

Martínez-Delgado D., Gabany R. J., Crawford K., et al., 2010, AJ, 140, 962

Mashchenko S., Couchman H. M. P., Wadsley J., 2006, Nature, 442, 539

Mayer L., Kazantzidis S., Madau P., Colpi M., Quinn T., Wadsley J., 2007, Science, 316, 1874

McCarthy I. G., Schaye J., Font A. S., Theuns T., Frenk C. S., Crain R. A., Dalla Vecchia C., 2012, MNRAS, submitted, arXiv: 1204.5195

McConnell N. J., Ma C.-P., Gebhardt K., Wright S. A., Murphy J. D., Lauer T. R., Graham J. R., Richstone D. O., 2011, Nature, 480, 215

McGaugh S. S., 2011, Physical Review Letters, 106, 121303

$-, 2012, \mathrm{AJ}, 143,40$

McLure R. J., Pearce H. J., Dunlop J. S., et al., 2012, MNRAS, submitted, arXiv:1205.4058

Milgrom M., 1983, ApJ, 270, 365

Monaghan J. J., 1992, ARA\&A, 30, 543

Moore B., Lake G., Katz N., 1998, ApJ, 495, 139

Navarro J. F., Steinmetz M., 2000, ApJ, 538, 477

Neistein E., Weinmann S. M., 2010, MNRAS, 405, 2717

Nickerson S., Stinson G., Couchman H. M. P., Bailin J., Wadsley J., 2011, MNRAS, 415, 257

Oh S.-H., Brook C., Governato F., Brinks E., Mayer L., de Blok W. J. G., Brooks A., Walter F., 2011, AJ, 142, 24

Okamoto T., 2012, MNRAS, submitted, arXiv: 1203.5372

Okamoto T., Gao L., Theuns T., 2008, MNRAS, 390, 920

Onions J., Knebe A., Pearce F. R., et al., 2012, MNRAS, 423, 1200

O'Shea B. W., Bryan G., Bordner J., Norman M. L., Abel T., Harkness R., Kritsuk A., 2005, in Lecture Notes in Computational Science and Engineering, Vol. 41, Adaptive Mesh Refinement - Theory and Applications, Plewa T., Linde T., Weirs V. G., eds., Springer, Berlin, Heidelberg, pp. 341-350, arXiv:astro-ph/0403044

Panter B., Jimenez R., Heavens A. F., Charlot S., 2007, MNRAS, 378, 1550

Peebles P. J. E., 2007, Nuovo Cimento B Serie, 122, 1035

Peirani S., Jung I., Silk J., Pichon C., 2012, MNRAS, submitted, arXiv:1205.4694

Peng Y.-j., Lilly S. J., Kovač K., et al., 2010, ApJ, 721, 193

Pérez-González P. G., Rieke G. H., Villar V., et al., 2008, ApJ, 675, 234

Pontzen A., Governato F., 2012, MNRAS, 421, 3464

Powell L. C., Slyz A., Devriendt J., 2011, MNRAS, 414, 3671

Press W. H., Schechter P., 1974, ApJ, 187, 425

Rees M. J., 1986, MNRAS, 218, 25P

Reines A. E., Deller A. T., 2012, ApJ, 750, L24

Rodighiero G., Daddi E., Baronchelli I., et al., 2011, ApJ, 739, L40

Salpeter E. E., 1955, ApJ, 121, 161

Salvadori S., Ferrara A., 2009, MNRAS, 395, L6

Sani E., Davies R. I., Sternberg A., et al., 2012, MNRAS, in press, arXiv:1205.4242

Scannapieco C., Wadepuhl M., Parry O. H., et al., 2012, MNRAS, 423, 1726

Schawinski K., 2012, in Frank N. Bash Symposium: New Horizons in Astronomy, arXiv:1206.2661 
Schawinski K., Thomas D., Sarzi M., Maraston C., Kaviraj S., Joo S.-J., Yi S. K., Silk J., 2007, MNRAS, 382,1415

Schechter P., 1976, ApJ, 203, 297

Sheth R. K., Mo H. J., Tormen G., 2001, MNRAS, 323, 1

Sijacki D., Springel V., Haehnelt M. G., 2009, MNRAS, 400, 100

Silk J., 1977, ApJ, 211, 638

-, 1997, ApJ, 481, 703

Silk J., Norman C., 2009, ApJ, 700, 262

Silk J., Nusser A., 2010, ApJ, 725, 556

Silk J., Rees M. J., 1998, A\&A, 331, L1

Somerville R. S., Hopkins P. F., Cox T. J., Robertson B. E., Hernquist L., 2008, MNRAS, 391, 481

Sonnenfeld A., Treu T., Gavazzi R., Marshall P. J., Auger M. W., Suyu S. H., Koopmans L. V. E., Bolton A. S., 2012, ApJ, 752, 163

Springel V., 2010a, MNRAS, 401, 791

- , 2010b, ARA\&A, 48, 391

Springel V., Hernquist L., 2003, MNRAS, 339, 312

Springel V., Wang J., Vogelsberger M., et al., 2008, MNRAS, 391, 1685

Springel V., White S. D. M., Jenkins A., et al., 2005, Nature, 435, 629

Stewart K. R., Kaufmann T., Bullock J. S., Barton E. J., Maller A. H., Diemand J., Wadsley J., 2011, ApJ, 735, L1

Sunyaev R. A., Zeldovich Y. B., 1972, Comments on Astrophysics and Space Physics, 4, 173

Swaters R. A., Sancisi R., van Albada T. S., van der Hulst J. M., 2011, ApJ, 729, 118

Teyssier R., 2002, A\&A, 385, 337

Teyssier R., Chapon D., Bournaud F., 2010, ApJ, 720, L149

Thomas D., Maraston C., Schawinski K., Sarzi M., Silk J., 2010, MNRAS, 404, 1775

Tikhonov A. V., Klypin A., 2009, MNRAS, 395, 1915

Tinker J., Kravtsov A. V., Klypin A., Abazajian K., Warren M., Yepes G., Gottlöber S., Holz D. E., 2008, ApJ, 688, 709

Tollerud E. J., Bullock J. S., Graves G. J., Wolf J., 2011, ApJ, 726, 108

Tolstoy E., Hill V., Tosi M., 2009, ARA\&A, 47, 371

Tremonti, C. A., Heckman, T. M., Kauffmann, G., et al., 2004, ApJ, 613, 898

Vale A., Ostriker J. P., 2006, MNRAS, 371, 1173

van der Wel A., Rix H.-W., Holden B. P., Bell E. F., Robaina A. R., 2009, ApJ, 706, L120

van Dokkum P. G., Conroy C., 2011, ApJ, 735, L13

Wagner A. Y., Bicknell G. V., 2011, ApJ, 728, 29

Wagner A. Y., Bicknell G. V., Umemura M., 2012, ApJ, submitted, arXiv:1205.0542

Wang R., Wagg J., Carilli C. L., et al., 2011, ApJ, 739, L34

Warren M. S., Abazajian K., Holz D. E., Teodoro L., 2006, ApJ, 646, 881

Weinmann S. M., Neistein E., Dekel A., 2011, MNRAS, 417, 2737

Weinmann S. M., Pasquali A., Oppenheimer B. D., Finlator K., Mendel J. T., Crain R. A., Maccio A. V., 2012, MNRAS, submitted, arXiv: 1204.4184

Weisz, D. R., Johnson, B. D., Johnson, L. C. et al., 2012, ApJ, 744, 44

Yang X., Mo H. J., van den Bosch F. C., 2003, MNRAS, 339, 1057

,- 2009, ApJ, 695, 900

Yoon J. H., Johnston K. V., Hogg D. W., 2011, ApJ, 731, 58

Ziegler B. L., Thomas D., Böhm A., Bender R., Fritz A., Maraston C., 2005, A\&A, 433, 519

Zolotov A., Brooks A. M., Willman B., et al., 2012, ApJ, submitted, arXiv:1207.0007 